\title{
Hybrid multisynchronization of coupled multistable memristive neural networks with time delays
}

\author{
Wei Yao ${ }^{\mathrm{a}}$, Chunhua Wang, ${ }^{\mathrm{a}, *}$, Jinde Cao ${ }^{\mathrm{b}}$, Yichuang Sun ${ }^{\mathrm{c}}$, Chao Zhou \\ ${ }^{a}$ College of Information Science and Engineering, Hunan University, Changsha, 410082, \\ China \\ ${ }^{b}$ School of Mathematics, Southeast University, Nanjing, 210096, China \\ ${ }^{c}$ School of Engineering and Technology, University of Hertfordshire, Hatfield AL10 9AB, \\ U.K.
}

\begin{abstract}
In this paper, we focus on synchronization issue of coupled multistable memristive neural networks (CMMNNs) with time delay under multiple stable equilibrium states. First, we build delayed CMMNNs consisting of one master subnetwork without controller and $N-1$ identical slave subnetworks with controllers, and every subnetwork has $n$ nodes. Moreover, this paper investigates multistability of delayed CMMNNs with continuous nonmonotonic piecewise linear activation function (PLAF) owning $2 r+2$ corner points. By using the theorems of differential inclusion and fixed point, sufficient conditions are derived such that master subnetwork of CMMNNs can acquire $(r+2)^{n}$ exponentially stable equilibrium points, stable periodic orbits or hybrid stable equilibrium states. Then, this paper proposes hybrid multisynchronization of delayed CMMNNs related with various external inputs under multiple stable equilibrium states for the first time. There exist $(r+2)^{n}$ hybrid multisynchronization manifolds in CMMNNs with different initial conditions and external inputs. Finally, two numerical simulations are given to illustrate the effectiveness of the obtained results.
\end{abstract}

\footnotetext{
Research supported in part by the National Natural Science Foundation of China (61571185), the Natural Science Foundation of Hunan Province, China (2016JJ2030) and the Science and Technology Planning Project of Hunan Province (2017GK4009).

* Corresponding author

Email addresses: wch1227164@hnu.edu.cn (Chunhua Wang )
}

Preprint submitted to Neurocomputing

June 20, 2019 
Key words: Memristive neural networks, controller, multistability, hybrid multisynchronization, external inputs.

\section{Introduction}

Memristor was first speculated by Chua in [1], and it was identified as the fourth basic circuit element. After memristor prototype was realized by HP Lab $[2]$, memristor-based circuits and applications $[3,4,5,6,7,8]$ have attracted in5 creasing attention. Using the nonvolatility of memristor $[7,8]$, conventional neural network (NN) system can be changed into memristive neural network (MNN) system by replacing resistor with memristor to emulate synapse. Because of the capability of memristor in storing and accessing data $[7,8]$ and the potential applications of MNN systems in many areas $[9,10,11]$ such as associative memory and static image processing, the dynamic characteristics of isolated MNN system have been widely studied, see [12, 13, 14, 15, 16, 17, 18, 19, 20, 21, 22]. Compared with an isolated system, coupled systems have wider applications in many fields $[23,24,25]$, such as robots, dynamic image processing, associative memory of video. Therefore, some dynamic characteristics of coupled systems

15 were investigated in recent years $[25,26,27,28,29,30,31,32,33,34,35,36]$. For example, synchronization of coupled NN systems was researched in $[30,31]$ and [35]. Papers [26, 27, 28, 29] studied synchronization of coupled MNN systems. In $[32,33,34]$, multistability and multisynchronization of coupled multistable NN systems were investigated. However, there is no relevant work on studying dynamic characteristics of coupled multistable MNN (CMMNN) systems.

As one of the most important dynamic characteristics, multistability of complex dynamical systems has been extensively investigated in recent years $[12,13,14]$, [37, 38, 39, 40, 41]. For example, papers [37, 38, 39, 40, 41] researched multistability of NNs. Wu and Zhang analyzed multistability of delayed MNNs with PLAF having 2 corner points in [13]. In [14], Nie et al. researched multistability of delayed MNNs with PLAF having 4 corner points. In the above researches $[13,14]$, MNNs can only obtain a small quantity of stable e- 
quilibrium states with a few corner points. Actually, as many stable equilibrium states as possible are very necessary for coupled systems including CMMNNs in some applications $[12,13,14],[23,24]$, [32, 33, 34], [36, 37, 38, 39, 40, 41, 42], such as associative memory storage, image processing.

Synchronization is a common phenomenon in nature [43], such as migratory birds, fireflies in the forest. And synchronization has broad potential applications in many areas [25], [44], such as secure communication, biological systems and so on. Over the years, there are many researches on studying synchronization problems $[15,16,17,18,19,20]$, [26, 27, 28, 29, 30, 31, 32, 33, 34, 35], $[45,46]$. For example, exponential synchronization of inertial BAM NNs [45], MNNs [15] and coupled MNNs [26] was researched. Asymptotical synchronization of MNNs [16] and coupled MNNs [27], anti-synchronization of MNNs

40 [17] were investigated. Zheng et al. studied finite-time projective synchronization of delayed fractional-order MNNs in [18]. Adaptive synchronization of MNNs in [19] and [20], lag synchronization of NNs [46] and coupled MNNs [28] were discussed. Li and Cao studied cluster synchronization of coupled stochastic NNs with time delay in [31]. In literatures $[15,16,17,18,19,20]$, ${ }_{45}[26,27,28,29,30,31],[45,46]$, synchronization was addressed under a stable equilibrium state. As discussed previously, multiple stable equilibrium states are very necessary for coupled systems including CMMNNs in some applications $[12,13,14],[23,24],[32,33,34],[36,37,38,39,40,41,42]$. Therefore, when coupled systems have multiple stable equilibrium states, how to achieve synchronization of coupled systems (called multisynchronization in this case) becomes more challenging and meaningful. During the last three years, multisynchronization of coupled multistable NN systems under multiple stable equilibrium states had aroused the interest of researchers. For instance, Wang et al. studied impulsive dynamical and static multisynchronization of delayed coupled multistable NNs in [32]. On the basis of [32], Zhang studied static multisynchronization of coupled multistable fractional-order NNs in [33] and Lv et al. investigated dynamical and static multisynchronization of coupled multistable NNs with parametric uncertainties in [34]. Literatures [32, 33, 34] achieved dy- 
namical (or static) multisynchronization by setting external inputs of all nodes as periodic (or constant) signals. However, these researches [32, 33, 34] neglect that external inputs of each node may be various in reality. Therefore, dynamical multisynchronization and static multisynchronization are not suitable for use in CMMNN systems when multiple stable equilibrium states exist in the systems and external inputs of each node are various.

Inspired by the aforementioned discussions, this paper focuses on synchronization issue of CMMNN systems. According to the literatures [32, 33, 34], it is necessary to ensure multistability in order to achieve synchronization of coupled multistable systems. Therefore, this paper studies multistability issue of CMMNN systems before achieving synchronization. To get a mass of stable equilibrium states, we extend the number of corner points of nonmonotonic PLAF to $2 r+2$ in this paper. In this case, the number of stable equilibrium states (stable equilibrium points, stable periodic orbits or hybrid stable equilibrium states) for the master subnetwork of CMMNNs is increased to $(r+2)^{n}$. To solve the above-mentioned problem which dynamical multisynchronization and static multisynchronization cannot solve, this paper proposes hybrid multisynchronization of CMMNNs related with various external inputs under multiple stable equilibrium states for the first time. Hybrid multisynchronization is a new type of synchronization phenomenon and has two features: owning multiple synchronization manifolds and considering various external inputs. It should be noted that dynamical and static multisynchronization introduced in $[32,33,34]$ can be seen as two special cases of hybrid multisynchronization. Combining various external inputs with the above-mentioned wide applications of memristor, coupled systems, multiple stable equilibrium states and synchronization, we can boldly speculate that hybrid multisynchronization of CMMNNs will have broad potential applications in some complex areas such as secure communication in multiple networks, obstacle avoidance for robots, formation flying of unmanned air vehicles and so on. Hence, the proposed results are general and meaningful, and improve the existing results.

The main contributions can be summarized as follows. 
1) This paper builds CMMNN systems which consist of one master subnetwork without controller and $N-1$ identical slave subnetworks with controllers, and every subnetwork has $n$ nodes. The special structure of CMMNNs makes it differ from drive-response (master-slave) system [15], [17], [19], coupled NNs [30, 31], [35], coupled MNNs [26, 27, 28], and coupled multistable NNs $[32,33,34]$. The advantages of this structure are that all slave subnetworks can synchronize the master subnetwork by controllers and multistability of the master subnetwork without controller can be addressed expediently.

2) The multistability of delayed CMMNNs is studied with continuous nonmonotonic PLAF owning $2 r+2$ corner points. By using theorems of differential inclusion and fixed point, sufficient conditions are derived such that the master subnetwork of CMMNNs has $(r+2)^{n}$ exponentially stable equilibrium points. Then on this basis, we study stable periodic orbits and hybrid stable equilibrium states (the hybrid of exponentially stable equilibrium points and stable periodic orbits), and obtain $(r+2)^{n}$ stable periodic orbits and $(r+2)^{n}$ hybrid stable equilibrium states. Compared with the existing researches [13, 14], this paper can obtain more stable equilibrium states.

3) Hybrid multisynchronization of delayed CMMNNs related with various external inputs under multiple stable equilibrium states is proposed for the first time. Hybrid multisynchronization can solve the problem that dynamical and static multisynchronization $[32,33,34]$ cannot take into consideration various external inputs. When some sufficient conditions are given, the CMMNNs with time delays can achieve hybrid multisynchronization and obtain $(r+2)^{n}$ hybrid multisynchronization manifolds. Both dynamical and static multisynchronization of delayed CMMNNs can also be achieved. It should be noted that dynamical and static multisynchronization introduced in [32, 33, 34] can be seen as two special cases of hybrid multisynchronization.

The rest of the paper is organized as follows. Some preliminaries are presented in Section 2. In Section 3, we build delayed CMMNNs with $(r+2)^{n}$ stable equilibrium states, and propose hybrid multisynchronization of delayed 
and 5 , respectively.

\section{Preliminaries}

First, we give some notations which will be used later.

Notations: $Z \leq 0$ represents that real matrix $Z$ is negative semidefinite, $Z \geq$ 0 represents that real matrix $Z$ is positive semidefinite. $C\left([-\tilde{\tau}, 0], \Re^{n}\right)$ denotes the space of continuous functions mapping $[-\tilde{\tau}, 0]$ into $\Re^{n} . P \otimes Q$ is Kronecker product of matrices $P$ and $Q . E_{m}$ is $m \times m$ unit matrix, [. . .] represents the interval. We define $\|\pi\|=\left(\sum_{i=1}^{n} \pi_{i}^{2}\right)^{\frac{1}{2}}$ for vector $\pi=\left(\pi_{1}, \pi_{2}, \ldots, \pi_{n}\right)^{T} \in \Re^{n} . \emptyset$ is empty set.

From [15],[17],[19], an isolated MNN with time delay can be considered as:

$$
\dot{x}(t)=-D x(t)+\Gamma(x(t)) f(x(t))+\mathrm{H}(x(t-\tau)) f(x(t-\tau))+I(t)
$$

where $x(t)=\left(x_{1}(t), x_{2}(t), \ldots, x_{n}(t)\right)^{T}$ represents state vector; $D=\operatorname{diag}\left\{d_{1}, d_{2}\right.$, $\left.\ldots, d_{n}\right\}$ is self-feedback vector for the corresponding nerve cells, $d_{i}>0, i=$ $1,2, \ldots, n ; \Gamma(x(t))=\left[\kappa_{i j}\left(x_{j}(t)\right)\right]_{n \times n}$ and $\mathrm{H}(x(t-\tau))=\left[\omega_{i j}\left(x_{j}\left(t-\tau_{i j}\right)\right)\right]_{n \times n} \mathrm{~s}-$ tand for memristive weight matrices; $f(x(t))=\left(f_{1}\left(x_{1}(t)\right), f_{2}\left(x_{2}(t)\right), \ldots, f_{n}\left(x_{n}(t)\right)\right)^{T}$ denotes activation function; $\tau_{i j}>0$ is time delay; $I(t)=\left(I_{1}(t), I_{2}(t), \ldots, I_{n}(t)\right)^{T}$ represents external input vector.

This paper considers two types of simple memristor models.

Memristor model (A) [19]:

$$
\kappa_{i j}\left(x_{j}(t)\right)= \begin{cases}\vec{\kappa}_{i j}, & \left|x_{j}(t)\right| \leq \xi_{j} \\ \overleftarrow{\kappa}_{i j}, & \left|x_{j}(t)\right|>\xi_{j}\end{cases}
$$

and

$$
\omega_{i j}\left(x_{j}\left(t-\tau_{i j}\right)\right)= \begin{cases}\vec{\omega}_{i j}, & \left|x_{j}\left(t-\tau_{i j}\right)\right| \leq \xi_{j}, \\ \overleftarrow{\omega}_{i j}, & \left|x_{j}\left(t-\tau_{i j}\right)\right|>\xi_{j},\end{cases}
$$

where $\vec{\kappa}_{i j}, \overleftarrow{\kappa}_{i j}, \vec{\omega}_{i j}$ and $\overleftarrow{\omega}_{i j}$ represent constants, $\xi_{j}>0$ is switching threshold.

Memristor model (B) [13]:

$$
\kappa_{i j}\left(x_{j}(t)\right)= \begin{cases}\vec{\kappa}_{i j}, & x_{j}(t) \leq 0 \\ \overleftarrow{\kappa}_{i j}, & x_{j}(t)>0\end{cases}
$$




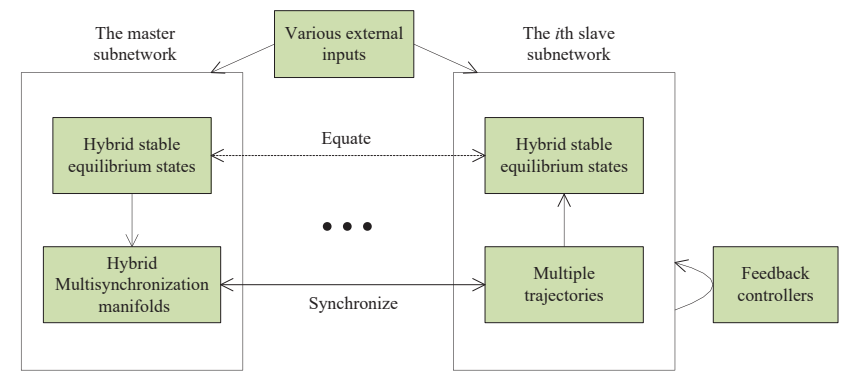

Figure 1: The schematic diagram of hybrid multisynchronization of CMMNNs.

and

$$
\omega_{i j}\left(x_{j}\left(t-\tau_{i j}\right)\right)= \begin{cases}\vec{\omega}_{i j}, & x_{j}\left(t-\tau_{i j}\right) \leq 0 \\ \overleftarrow{\omega}_{i j}, & x_{j}\left(t-\tau_{i j}\right)>0 .\end{cases}
$$

Define a set of positive integers $\mathrm{Z}=\{1,2, \ldots, n\}$, let $U$ and $V$ as two subsets of Z, and satisfy the following relationship: $U \cap V=\emptyset$ and $U \cup$ $V=\mathrm{Z}$. Define three sets of external inputs $\vartheta=\left\{I_{i}(t)\right.$, for all $\left.i \in \mathrm{Z}\right\}, \Omega=$ $\left\{I_{u}(t)\right.$, for all $\left.u \in U\right\}, \Lambda=\left\{I_{v}(t)\right.$, for all $\left.v \in V\right\}$, then we can get $\Omega \cap \Lambda=\emptyset$ and $\Omega \cup \Lambda=\vartheta$. $I_{u}(t)$ is periodical input, that is, $I_{u}\left(t+T_{u}\right)=I_{u}(t)$, for all $u \in U ; I_{v}(t)$ is constant, i.e., $I_{v}(t)=I_{v}$, for all $v \in V$.

\section{Main Results}

This paper presents the schematic diagram of hybrid multisynchronization of CMMNNs, as shown in Fig. 1. First of all, we need to ensure that the master subnetwork of CMMNNs can obtain $(r+2)^{n}$ hybrid stable equilibrium states with various external inputs. It means that there are $(r+2)^{n}$ hybrid multisynchronization manifolds. Then multiple trajectories of every slave subnetwork can achieve synchronization with hybrid multisynchronization manifolds of the master subnetwork via feedback controllers and various external inputs. Therefore, every slave subnetwork can also obtain $(r+2)^{n}$ hybrid stable equilibrium states. 


\section{1. $C M M N N s$} subnetwork in this paper does not have controller. In addition, compared with drive-response or master-slave NNs introduced in [15], [17] and [19], delayed CMMNNs own more subnetworks and more complex dynamic behaviors.

We consider delayed CMMNNs with mathematical formula as follows:

$$
\left\{\begin{array}{c}
\dot{x}_{i}(t)=-D x_{i}(t)+\Gamma\left(x_{i}(t)\right) f\left(x_{i}(t)\right)+\mathrm{H}\left(x_{i}(t-\tau)\right) \\
\times f\left(x_{i}(t-\tau)\right)+I_{i}(t)+\diamond_{i}(t), i=1,2, \ldots, N-1 ; \\
\dot{x}_{N}(t)=-D x_{N}(t)+\Gamma\left(x_{N}(t)\right) f\left(x_{N}(t)\right)+\mathrm{H}\left(x_{N}(t-\tau)\right) \\
\times f\left(x_{N}(t-\tau)\right)+I_{N}(t)
\end{array}\right.
$$

where the variables and parameters are the same as those given by $(1) ; \diamond_{i}(t)=$ 165 $\left(\diamond_{i 1}(t), \diamond_{i 2}(t), \ldots, \diamond_{i n}(t)\right)^{T}, i=1,2, \ldots, N-1$, represent controllers; $I_{i}(t)$ $=\left(I_{i 1}(t), I_{i 2}(t), \ldots, I_{i n}(t)\right)^{T} ; I_{i k}(t)=I_{j k}(t)$ for $i, j=1,2, \ldots, N$ and $k=$ $1,2, \ldots, n . x_{i}(t), i=1, \ldots, N-1$, are $N-1$ identical slave subnetworks; $x_{N}(t)$ $=\left(x_{N 1}(t), x_{N 2}(t), \ldots, x_{N n}(t)\right)^{T}$ represents the master subnetwork. It is obvious that the structure of master subnetwork is the same as the isolated MNN with time delay (1).

Remark 1: Due to the simple structure of master subnetwork, it is easy to research and analyse dynamic behaviors of CMMNNs through master subnetwork, such as multistability. So, it can reduce operation time and cost.

\subsection{Multistability of delayed CMMNNs}

To increase the number of stable equilibrium states (equilibrium points or periodic orbits) of CMMNNs, this paper considers a class of continuous non- 
monotonic PLAF as

$$
f_{i}(s)=\left\{\begin{array}{cc}
u_{i}, & s \in\left(-\infty, p_{i}^{0}\right] \\
k_{i}^{0} s+m_{i}^{0}, & s \in\left(p_{i}^{0}, q_{i}^{0}\right) \\
l_{i}^{0} s+n_{i}^{0}, & s \in\left[q_{i}^{0}, p_{i}^{1}\right] \\
\vdots & \vdots \\
k_{i}^{r} s+m_{i}^{r}, & s \in\left(p_{i}^{r}, q_{i}^{r}\right) \\
v_{i}, & s \in\left[q_{i}^{r},+\infty\right)
\end{array}\right.
$$

175 constants and $u_{i}<v_{i} ; k_{i}^{j} \geq 0 ; l^{j} \leq 0 ; p_{i}^{0}<q_{i}^{0}<p_{i}^{1}<\ldots<q_{i}^{r} ; \min \left\{f_{i}(s)\right\}=u_{i}$ and $\max \left\{f_{i}(s)\right\}=v_{i}$.

Remark 2: Obviously, continuous nonmonotonic $\operatorname{PLAF}(3): f_{i}(s), i=$ $1,2, \ldots, n$ satisfy Lipschitz condition: $\left|f_{i}(\wedge)-f_{i}(\vee)\right| \leq l_{i}|\wedge-\vee|$ for any $\wedge$, ${ }_{180} \vee \in \Re$, where $l_{i}=\max \left\{k_{i}^{0}, k_{i}^{1}, \ldots, k_{i}^{r},\left|l_{i}^{0}\right|,\left|l_{i}^{1}\right|, \ldots,\left|l_{i}^{r-1}\right|\right\}$. Meanwhile, PLAF (3) is bounded, i.e. there exist constant $\mu_{i}=\max \left\{\left|u_{i}\right|,\left|v_{i}\right|\right\}$, so that $\left|f_{i}(a)\right| \leq \mu_{i}$ for any $a \in \Re$.

We denote

$$
\begin{gathered}
\left(-\infty, p_{i}^{0}\right]=\left(-\infty, p_{i}^{0}\right]^{1} \times\left(p_{i}^{0}, q_{i}^{0}\right)^{0} \times \cdots \times\left[q_{i}^{r},+\infty\right)^{0}, \\
\left(p_{i}^{0}, q_{i}^{0}\right)=\left(-\infty, p_{i}^{0}\right]^{0} \times\left(p_{i}^{0}, q_{i}^{0}\right)^{1} \times \cdots \times\left[q_{i}^{r},+\infty\right)^{0}, \\
\cdots \\
{\left[q_{i}^{r},+\infty\right)=\left(-\infty, p_{i}^{0}\right]^{0} \times\left(p_{i}^{0}, q_{i}^{0}\right)^{0} \times \cdots \times\left[q_{i}^{r},+\infty\right)^{1} .}
\end{gathered}
$$

Then $\Re^{n}$ is divided into $(2 r+3)^{n}$ parts, that is

$$
\begin{gathered}
\Psi=\left\{\prod_{i=1}^{n}\left(-\infty, p_{i}^{0}\right]^{\lambda_{i}^{1}} \times\left(p_{i}^{0}, q_{i}^{0}\right)^{\lambda_{i}^{2}} \times \cdots \times\left[q_{i}^{r},+\infty\right)^{\lambda_{i}^{2 r+3}},\right. \\
\left(\lambda_{i}^{1}, \lambda_{i}^{2}, \cdots, \lambda_{i}^{2 r+3}\right)=(1,0, \cdots, 0) \text { or }(0,1, \cdots, 0) \text { or } \\
\cdots \operatorname{or}(0,0, \cdots, 1)\} . \\
\text { Let } \tilde{\kappa}_{i j}=\max \left\{\left|\vec{\kappa}_{i j}\right|,\left|\overleftarrow{\kappa}_{i j}\right|\right\}, \tilde{\omega}_{i j}=\max \left\{\left|\vec{\omega}_{i j}\right|,\left|\overleftarrow{\omega}_{i j}\right|\right\}, \bar{\kappa}_{i j}=\max \left\{\vec{\kappa}_{i j}, \overleftarrow{\kappa}_{i j}\right\}, \\
\breve{\kappa}_{i j}=\min \left\{\vec{\kappa}_{i j}, \overleftarrow{\kappa}_{i j}\right\}, \bar{\omega}_{i j}=\max \left\{\vec{\omega}_{i j}, \overleftarrow{\omega}_{i j}\right\}, \breve{\omega}_{i j}=\min \left\{\vec{\omega}_{i j}, \overleftarrow{\omega}_{i j}\right\} . \text { Set } L_{i}= \\
\max \left\{0,\left|l_{i}^{0}\right|,\left|l_{i}^{1}\right|, \ldots,\left|l_{i}^{r-1}\right|\right\} \text { for } i=1,2, \ldots, n .
\end{gathered}
$$


For a given set $\mathrm{X} \subset \Re, c o[\mathrm{X}]$ indicates the closure of the convex hull for $\mathrm{X}$. Therefore, by memristor model (A), we can get

$$
\begin{gathered}
c o\left[\kappa_{i j}\left(x_{j}(t)\right)\right]=\left\{\begin{array}{cl}
\vec{\kappa}_{i j}, & \left|x_{j}(t)\right|<\xi_{j}, \\
{\left[\widetilde{\kappa}_{i j}, \bar{\kappa}_{i j}\right],} & \left|x_{j}(t)\right|=\xi_{j}, \\
\overleftarrow{\kappa}_{i j}, & \left|x_{j}(t)\right|>\xi_{j} .
\end{array}\right. \\
c o\left[\omega_{i j}\left(x_{j}\left(t-\tau_{i j}\right)\right)\right]=\left\{\begin{array}{cl}
\vec{\omega}_{i j}, & \left|x_{j}\left(t-\tau_{i j}\right)\right|<\xi_{j}, \\
{\left[\bar{\omega}_{i j}, \bar{\omega}_{i j}\right],} & \left|x_{j}\left(t-\tau_{i j}\right)\right|=\xi_{j}, \\
\overleftarrow{\omega}_{i j}, & \left|x_{j}\left(t-\tau_{i j}\right)\right|>\xi_{j} .
\end{array}\right.
\end{gathered}
$$

According to the theory of differential inclusions, we can rewrite the master subnetwork of CMMNNs (2) as

$$
\dot{x}(t) \in-D x(t)+c o[\Gamma(x(t))] f(x(t))+c o[\mathrm{H}(x(t-\tau))] f(x(t-\tau))+I(t),
$$

where $c o[\Gamma(x(t))]=\left[c o\left[\kappa_{i j}\left(x_{j}(t)\right)\right]\right]_{n \times n}, c o[\mathrm{H}(x(t-\tau))]=\left[c o\left[\omega_{i j}\left(x_{j}\left(t-\tau_{i j}\right)\right)\right]\right]_{n \times n}$, $x(t)=x_{N}(t)=\left(x_{N 1}(t), x_{N 2}(t), \ldots, x_{N n}(t)\right)^{T}$.

So, there exist $\hat{\kappa}_{i j}\left(x_{j}(t)\right) \in c o\left[\kappa_{i j}\left(x_{j}(t)\right)\right], \hat{\omega}_{i j}\left(x_{j}\left(t-\tau_{i j}\right)\right) \in c o\left[\omega_{i j}\left(x_{j}\left(t-\tau_{i j}\right)\right)\right]$, such that

$$
\dot{x}(t)=-D x(t)+\hat{\Gamma}(x(t)) f(x(t))+\hat{\mathrm{H}}(x(t-\tau)) f(x(t-\tau))+I(t)
$$

where $\hat{\Gamma}(x(t))=\left[\hat{\kappa}_{i j}\left(x_{j}(t)\right)\right]_{n \times n}$ and $\hat{\mathrm{H}}(x(t-\tau))=\left[\hat{\omega}_{i j}\left(x_{j}\left(t-\tau_{i j}\right)\right)\right]_{n \times n}$.

Lemma 1 [19]: For PLAF (3) and memristor model (A), if $f_{j}\left( \pm \xi_{j}\right)=0$, $j=1,2, \ldots, n$, we have

$$
\begin{aligned}
& \left|c o\left[\kappa_{i j}\left(x_{j}(t)\right)\right] f_{j}\left(x_{j}(t)\right)-c o\left[\kappa_{i j}\left(y_{j}(t)\right)\right] f_{j}\left(y_{j}(t)\right)\right| \\
& \leq \tilde{\kappa}_{i j} l_{j}\left|x_{j}(t)-y_{j}(t)\right|, \quad i, j=1,2, \ldots, n .
\end{aligned}
$$

Theorem 1: The master subnetwork of CMMNNs (2) can have $(r+2)^{n}$ exponentially stable equilibrium points in $\Re^{n}$ with PLAF (3) and memristor model (A), if $\Omega=\emptyset, \Lambda=\vartheta, f_{i}\left( \pm \xi_{i}\right)=0$ and

$$
\begin{aligned}
& -d_{i} p_{i}^{c}+\Upsilon_{i 1}+\Upsilon_{i 2}+\Upsilon_{i 3}+I_{i}<0, \\
& -d_{i} q_{i}^{c}+\Upsilon_{i 4}+\Upsilon_{i 5}+\Upsilon_{i 6}+I_{i}>0,
\end{aligned}
$$


hold for $i=1,2, \ldots, n$ and $c=1,2, \ldots, r$, where

$$
\begin{gathered}
\Upsilon_{i 1}=\max \left\{\breve{\kappa}_{i i} f_{i}\left(p_{i}^{c}\right), \bar{\kappa}_{i i} f_{i}\left(p_{i}^{c}\right)\right\}, \\
\Upsilon_{i 2}=\sum_{j=1, j \neq i}^{n} \max \left\{\breve{\kappa}_{i j} u_{j}, \bar{\kappa}_{i j} u_{j}, \breve{\kappa}_{i j} v_{j}, \bar{\kappa}_{i j} v_{j}\right\}, \\
\Upsilon_{i 3}=\sum_{j=1}^{n} \max \left\{\breve{\omega}_{i j} u_{j}, \bar{\omega}_{i j} u_{j}, \breve{\omega}_{i j} v_{j}, \bar{\omega}_{i j} v_{j}\right\}, \\
\Upsilon_{i 4}=\min \left\{\breve{\kappa}_{i i} f_{i}\left(q_{i}^{c}\right), \bar{\kappa}_{i i} f_{i}\left(q_{i}^{c}\right)\right\}, \\
\Upsilon_{i 6}=\sum_{j=1, j \neq i}^{n} \min \left\{\breve{\kappa}_{i j} u_{j}, \bar{\kappa}_{i j} u_{j}, \breve{\kappa}_{i j} v_{j}, \bar{\kappa}_{i j} v_{j}\right\}, \\
\min \left\{\breve{\omega}_{i j} u_{j}, \bar{\omega}_{i j} u_{j}, \breve{\omega}_{i j} v_{j}, \bar{\omega}_{i j} v_{j}\right\},
\end{gathered}
$$

meanwhile, the following inequation:

$$
d_{i}-\sum_{j=1}^{n} \tilde{\kappa}_{i j} L_{j}-\sum_{j=1}^{n} \tilde{\omega}_{i j} L_{j}>0, \quad i=1,2, \ldots, n,
$$

holds.

Proof: See Appendix A.

Remark 3: Paper [39] researched multistability of delayed NNs with PLAF. $(2 k)^{n}$ exponentially stable equilibrium points can be obtained by sufficient condition. However, each corner point of PLAF is fixed, which can be set free in later researches. Compared with conventional NNs used in [39], MNNs are practical and have complex dynamic behaviors.

Remark 4: It is shown from [13] that delayed MNNs with $n$ nodes and PLAF owning 2 corner points can get $2^{n}$ exponentially stable equilibrium points. In [14], Nie et al. researched that nonmonotonic PLAF with four corner points can obtain $3^{n}$ locally stable equilibria for delayed MNNs. In this paper, corner points of PLAF are extended from 2 and 4 to $2 r+2$. In the meanwhile, the number of exponentially stable equilibrium points is increased to $(r+2)^{n}$. For all we know, there is little work on multistability of delayed CMMNNs with continuous nonmonotonic PLAF owning $2 r+2$ corner points. 
When $\Omega$ and $\Lambda$ satisfy different conditions, this paper can get corollaries 1 and 2 .

Corollary 1: The master subnetwork of CMMNNs (2) can obtain $(r+2)^{n}$ stable periodic orbits in $\Re^{n}$ with PLAF (3) and memristor model (A), if $\Omega=\vartheta$, $\Lambda=\emptyset, f_{i}\left( \pm \xi_{i}\right)=0$ and

$$
\begin{gathered}
-d_{i} p_{i}^{c}+\Upsilon_{i 1}+\Upsilon_{i 2}+\Upsilon_{i 3}+I_{i}(t)<0, \\
-d_{i} q_{i}^{c}+\Upsilon_{i 4}+\Upsilon_{i 5}+\Upsilon_{i 6}+I_{i}(t)>0, \\
d_{i}-\sum_{j=1}^{n} \tilde{\kappa}_{i j} L_{j}-\sum_{j=1}^{n} \tilde{\omega}_{i j} L_{j}>0,
\end{gathered}
$$

hold for $c=0,1, \ldots, r$ and $i=1, \ldots, n$.

Proof: According to lemma 1 in [32], the master subnetwork of CMMNNs (2) can have $(r+2)^{n}$ stable periodic orbits.

Corollary 2: The master subnetwork of CMMNNs (2) can obtain $(r+2)^{n}$ hybrid stable equilibrium states in $\Re^{n}$ with PLAF (3) and memristor model (A), if $\Omega \neq \emptyset, \Lambda \neq \emptyset, f_{i}\left( \pm \xi_{i}\right)=0$ and the following conditions:

$$
\begin{gathered}
-d_{u} p_{u}^{c}+\Upsilon_{u 1}+\Upsilon_{u 2}+\Upsilon_{u 3}+I_{u}(t)<0, \\
-d_{u} q_{u}^{c}+\Upsilon_{u 4}+\Upsilon_{u 5}+\Upsilon_{u 6}+I_{u}(t)>0, \\
-d_{v} p_{v}^{c}+\Upsilon_{v 1}+\Upsilon_{v 2}+\Upsilon_{v 3}+I_{v}<0, \\
-d_{v} q_{v}^{c}+\Upsilon_{v 4}+\Upsilon_{v 5}+\Upsilon_{v 6}+I_{v}>0, \\
d_{i}-\sum_{j=1}^{n} \tilde{\kappa}_{i j} L_{j}-\sum_{j=1}^{n} \tilde{\omega}_{i j} L_{j}>0,
\end{gathered}
$$

hold for all $u \in U$, all $v \in V, c=0,1, \ldots, r$ and $i=1, \ldots, n$.

Proof: $\Omega=\left\{I_{u}(t)\right.$, for all $\left.u \in U\right\}$ is nonempty set, it means that $x_{N u}(t)$ for all $u \in U$ will appear stable periodic orbit as time $t \rightarrow \infty$ according to the lemma 1 in [32]. Similarly, $\Lambda=\left\{I_{v}\right.$, for all $\left.v \in V\right\}$ is nonempty set, it means that $x_{N v}(t)$ for all $v \in V$ will appear exponentially stable equilibrium point as time $t \rightarrow \infty$ according to the theorem 1 . Therefore, the master subnetwork of 
By set-valued maps and memristor model (B), we can get

$$
\begin{gathered}
c o\left[\kappa_{i j}\left(x_{j}(t)\right)\right]=\left\{\begin{array}{cl}
\vec{\kappa}_{i j}, & x_{j}(t)<0, \\
{\left[\widetilde{\kappa}_{i j}, \bar{\kappa}_{i j}\right],} & x_{j}(t)=0, \\
\overleftarrow{\kappa}_{i j}, & x_{j}(t)>0 .
\end{array}\right. \\
c o\left[\omega_{i j}\left(x_{j}\left(t-\tau_{i j}\right)\right)\right]=\left\{\begin{array}{cl}
\vec{\omega}_{i j} & x_{j}\left(t-\tau_{i j}\right)<0, \\
{\left[\bar{\omega}_{i j}, \bar{\omega}_{i j}\right],} & x_{j}\left(t-\tau_{i j}\right)=0, \\
\overleftarrow{\omega}_{i j} & x_{j}\left(t-\tau_{i j}\right)>0 .
\end{array}\right.
\end{gathered}
$$

Now, PLAF (3) is simplified to the following form

$$
f_{i}(s)=\left\{\begin{array}{cc}
u_{i}, & s \in\left(-\infty, p_{i}^{0}\right] \\
\beta_{i}^{0} s, & s \in\left(p_{i}^{0}, q_{i}^{0}\right) \\
v_{i}, & s \in\left[q_{i}^{0},+\infty\right)
\end{array}\right.
$$

Obviously, $f_{i}(0)=0, L_{i}=0, i=1, \ldots, n,(6)$ holds. From PLAF (12) and memristor model (B), we can have

$$
\begin{aligned}
& \left|c o\left[\kappa_{i j}\left(x_{j}(t)\right)\right] f_{j}\left(x_{j}(t)\right)-c o\left[\kappa_{i j}\left(y_{j}(t)\right)\right] f_{j}\left(y_{j}(t)\right)\right| \\
& \leq \tilde{\kappa}_{i j} \beta_{j}^{0}\left|x_{j}(t)-y_{j}(t)\right|=\tilde{\kappa}_{i j} l_{j}\left|x_{j}(t)-y_{j}(t)\right| .
\end{aligned}
$$

where $i$ and $j=1, \ldots, n$.

Then, this paper can get the following three corollaries.

Corollary 3: The master subnetwork of CMMNNs (2) can get $2^{n}$ exponentially stable equilibrium points in $\Re^{n}$ with PLAF (12) and memristor model (B), if $\Omega=\emptyset, \Lambda=\vartheta$, and

$$
\begin{aligned}
& -d_{i} p_{i}^{0}+\Upsilon_{i 1}^{*}+\Upsilon_{i 2}+\Upsilon_{i 3}+I_{i}<0, \\
& -d_{i} q_{i}^{0}+\Upsilon_{i 4}^{*}+\Upsilon_{i 5}+\Upsilon_{i 6}+I_{i}>0,
\end{aligned}
$$

hold for $i=1, \ldots, n$, where

$$
\begin{aligned}
& \Upsilon_{i 1}^{*}=\max \left\{\breve{\kappa}_{i i} f_{i}\left(p_{i}^{0}\right), \bar{\kappa}_{i i} f_{i}\left(p_{i}^{0}\right)\right\}, \\
& \Upsilon_{i 4}^{*}=\min \left\{\breve{\kappa}_{i i} f_{i}\left(q_{i}^{0}\right), \bar{\kappa}_{i i} f_{i}\left(q_{i}^{0}\right)\right\} .
\end{aligned}
$$


Corollary 4: The master subnetwork of CMMNNs (2) can get $2^{n}$ stable periodic orbits in $\Re^{n}$ with PLAF (12) and memristor model (B), if $\Omega=\vartheta$, $\Lambda=\emptyset$, and

$$
\begin{aligned}
& -d_{i} p_{i}^{0}+\Upsilon_{i 1}^{*}+\Upsilon_{i 2}+\Upsilon_{i 3}+I_{i}(t)<0, \\
& -d_{i} q_{i}^{0}+\Upsilon_{i 4}^{*}+\Upsilon_{i 5}+\Upsilon_{i 6}+I_{i}(t)>0
\end{aligned}
$$

hold for $i=1, \ldots, n$.

Corollary 5: The master subnetwork of CMMNNs (2) can get $2^{n}$ hybrid stable equilibrium states in $\Re^{n}$ with PLAF (12) and memristor model (B), if $\Omega \neq \emptyset, \Lambda \neq \emptyset$, and

$$
\begin{gathered}
-d_{u} p_{u}^{0}+\Upsilon_{u 1}^{*}+\Upsilon_{u 2}+\Upsilon_{u 3}+I_{u}(t)<0 \\
-d_{u} q_{u}^{0}+\Upsilon_{u 4}^{*}+\Upsilon_{u 5}+\Upsilon_{u 6}+I_{u}(t)>0 \\
-d_{v} p_{v}^{0}+\Upsilon_{v 1}^{*}+\Upsilon_{v 2}+\Upsilon_{v 3}+I_{v}<0 \\
-d_{v} q_{v}^{0}+\Upsilon_{v 4}^{*}+\Upsilon_{v 5}+\Upsilon_{v 6}+I_{v}>0
\end{gathered}
$$

hold for all $u \in U$, all $v \in V$.

\subsection{Hybrid Multisynchronization}

First, we present two necessary definitions.

Definition 1: $\operatorname{HMSM}(t)$ is called hybrid multisynchronization manifold of CMMNNs with time delays (2), if $H M S M(t)=x_{N}(t)=\left(x_{N 1}(t), x_{N 2}(t), \ldots\right.$, $\left.x_{N n}(t)\right)^{T} \in \Re^{n}$ and the following conditions hold.

1) $x_{i j}(t) \rightarrow x_{N j}(t)$ as $t \rightarrow \infty$ for any $i=1, \ldots, N-1, j=1, \ldots, n$.

2) For $x_{N k}(t), k=1, \ldots, n$, it is either stable periodic orbit or stable equilibrium point as $t \rightarrow \infty$.

3) Stable periodic orbit and stable equilibrium point coexist in $x_{N}(t)$ simultaneously.

230 Definition 2: The delayed CMMNNs (2) can be said to achieve hybrid multisynchronization when the following conditions hold.

1) Sets $\Omega \neq \emptyset, \Lambda \neq \emptyset$.

2) Given arbitrary initial values $x\left(t_{0}\right)=\left(x_{1}\left(t_{0}\right)^{T}, x_{2}\left(t_{0}\right)^{T}, \ldots, x_{N}\left(t_{0}\right)^{T}\right)^{T}$, where $x_{i}\left(t_{0}\right) \in C\left([-\tilde{\tau}, 0], \Re^{n}\right)$ for $i=1, \ldots, N$, and $\tilde{\tau}=\max _{1 \leq i \leq n, 1 \leq j \leq n} \tau_{i j}$, then 
there exist hybrid multisynchronization manifold $H M S M_{w}(t)=x_{N}(t)=\left(x_{N 1}(t)\right.$, $\left.x_{N 2}(t), \ldots, x_{N n}(t)\right)^{T} \in \Re^{n}, \gamma>0$, and $Y>0$, such that

$$
\left\|x_{i}(t)-H M S M_{w}(t)\right\|=\left\|x_{i}(t)-x_{N}(t)\right\| \leq Y e^{-\gamma t}
$$

for any $t \geq 0, i=1, \ldots, N-1$, subscript $w$ is certain positive integer.

3) There exist at least two different hybrid multisynchronization manifolds ${ }_{235} H M S M_{w}(t)$ and $H M S M_{y}(t)$ with the corresponding different initial values $x\left(t_{0}\right)$ and $x^{\prime}\left(t_{0}\right)$.

Remark 5: For $x_{N k}(t)$, it is either stable periodic orbit or stable equilibrium point related with corresponding external input $I_{N k}(t), k=1,2, \ldots, n$, as time $t \rightarrow \infty$, that is, if $I_{N k}(t) \in \Omega, x_{N k}(t)$ is stable periodic orbit, else $I_{N k}(t) \in \Lambda$, ${ }_{240} x_{N k}(t)$ will be stable equilibrium point. It should be emphasized, $x_{N}(t)=$ $\left(x_{N 1}(t), x_{N 2}(t), \ldots, x_{N n}(t)\right)^{T}$ are hybrid stable equilibrium states. Therefore, hybrid multisynchronization manifolds $H_{M S M}(t)$ and $H_{M S M}(t)$ are also hybrid stable equilibrium states. According to corollary 2, the master subnetwork of CMMNNs (2) can have $(r+2)^{n}$ hybrid stable equilibrium states in $\Re^{n}$. It means that there are $(r+2)^{n}$ hybrid multisynchronization manifolds in CMMNNs (2).

When coupled systems have multiple stable equilibrium states, we call the synchronization of coupled systems as multisynchronization. In other word, there exist multiple trajectories for every subnetwork of coupled systems. When there exist only multiple stable equilibrium points (or stable periodic orbits), the multisynchronization of coupled systems is static (or dynamical). During the last three years, dynamical and static multisynchronization were addressed in $[32,33,34]$. When all stable equilibrium states are hybrid (namely, the hybrid of stable equilibrium points and stable periodic orbits), the multisynchronization of coupled systems is called hybrid multisynchronization.

To achieve hybrid multisynchronization of delayed CMMNNs, we design con- 
trollers of slave subnetworks as follows:

$$
\begin{gathered}
\diamond_{i}(t)=\sum_{j=1}^{N} \eta_{i j} \Xi x_{j}(t)+\sum_{j=1}^{N} \sigma_{i j} \Xi \varphi\left(x_{j}(t)-x_{i}(t)\right), \\
i=1,2, \ldots, N-1,
\end{gathered}
$$

where coupling matrix $\Xi=\operatorname{diag}\left\{\Xi_{1}, \Xi_{2}, \ldots, \Xi_{n}\right\} \geq 0 ; \eta_{i j}$ and $\sigma_{i j}$ represent coupling strength. We define that $\sigma_{i j} \geq 0$ for $i \neq j$ and $\sigma_{i j}=0$ for $i=j$. $\varphi\left(x_{j}(t)-x_{i}(t)\right)=\left(\varphi\left(x_{j 1}(t)-x_{i 1}(t)\right), \varphi\left(x_{j 2}(t)-x_{i 2}(t)\right), \ldots, \varphi\left(x_{j n}(t)-x_{i n}(t)\right)\right)^{T}$ denotes nonlinear coupling function, and we set

$$
\varphi\left(x_{j}(t)-x_{i}(t)\right)=\operatorname{sgn}\left(x_{j}(t)-x_{i}(t)\right)
$$

where sgn represents sign function.

Therefore, delayed CMMNNs (2) can be written as:

$$
\begin{aligned}
\dot{x}(t) & =-\left(E_{N} \otimes D\right) x(t)+\left(E_{N} \otimes \Gamma(x(t))\right) f(x(t)) \\
& +\left(E_{N} \otimes \mathrm{H}(x(t-\tau))\right) f(x(t-\tau))+\bar{I}(t)+(\Sigma \otimes \Xi) x(t)+\Theta,
\end{aligned}
$$

where $x(t)=\left(x_{1}(t)^{T}, x_{2}(t)^{T}, \ldots, x_{N}(t)^{T}\right)^{T}, x_{i}(t)=\left(x_{i 1}(t), x_{i 2}(t), \ldots, x_{i n}(t)\right)^{T}$ for $i=1, \ldots, N . f(x(t))=\left(f\left(x_{1}(t)\right)^{T}, f\left(x_{2}(t)\right)^{T}, \ldots, f\left(x_{N}(t)\right)^{T}\right)^{T}, f\left(x_{i}(t)\right)=$ $\left(f_{1}\left(x_{i 1}(t)\right), f_{2}\left(x_{i 2}(t)\right), \ldots, f_{n}\left(x_{i n}(t)\right)\right)^{T}, i=1, \ldots, N . \bar{I}(t)=\left(I_{1}(t)^{T}, I_{2}(t)^{T}, \ldots\right.$, $\left.I_{N}(t)^{T}\right)^{T}, \Sigma=\left[\eta_{i j}\right]_{N \times N}, \eta_{N j}=0$ for $j=1, \ldots, N$.

$$
\Theta=\left[\begin{array}{c}
\sum_{j=1}^{N} \sigma_{1 j} \Xi \operatorname{sgn}\left(x_{j}(t)-x_{1}(t)\right) \\
\vdots \\
\sum_{j=1}^{N} \sigma_{(N-1) j} \Xi \operatorname{sgn}\left(x_{j}(t)-x_{N-1}(t)\right) \\
0
\end{array}\right] .
$$

We define synchronization error as $e_{i}(t)=x_{i}(t)-x_{N}(t), i=1, \ldots, N-1$. Therefore, we get

$$
\begin{aligned}
& \dot{e}_{i}(t)=-D e_{i}(t)+\Gamma\left(x_{i}(t)\right) f\left(x_{i}(t)\right)-\Gamma\left(x_{N}(t)\right) f\left(x_{N}(t)\right) \\
& +\mathrm{H}\left(x_{i}(t-\tau)\right) f\left(x_{i}(t-\tau)\right)-\mathrm{H}\left(x_{N}(t-\tau)\right) f\left(x_{N}(t-\tau)\right) \\
& +\left(\Sigma_{i} \otimes \Xi\right) x(t)+\Theta_{i}, \quad i=1,2, \ldots, N-1,
\end{aligned}
$$

where $\Sigma_{i}=\left(\eta_{i 1}, \eta_{i 2}, \ldots, \eta_{i N}\right), \Theta_{i}=\sum_{j=1}^{N} \sigma_{i j} \Xi \operatorname{sgn}\left(x_{j}(t)-x_{i}(t)\right)$. 
We define a matrix $W=\left(w_{i j}\right)_{(N-1) \times N}$, and

$$
w_{i j}=\left\{\begin{array}{cc}
1, & i=j \\
-1, & j=N \\
0, & \text { others }
\end{array}\right.
$$

Set $\tilde{W}=W \otimes E_{n}$, then $e(t)=\tilde{W} x(t)$, where $e(t)=\left(e_{1}(t)^{T}, e_{2}(t)^{T}, \ldots\right.$, $\left.e_{N-1}(t)^{T}\right)^{T}$. Let $\hat{P}=\operatorname{diag}\left\{\hat{p}_{1}, \hat{p}_{2}, \ldots, \hat{p}_{n}\right\}, \hat{Q}=\operatorname{diag}\left\{\hat{q}_{1}, \hat{q}_{2}, \ldots, \hat{q}_{n}\right\}$, and de${ }_{260}$ note $\tilde{P}=E_{N-1} \otimes \hat{P}, \varepsilon_{i}=\sigma_{i N}-\sum_{j=1}^{N-1} \sigma_{i j}, i=1, \ldots, N-1, \tilde{Q}=E_{N-1} \otimes \hat{Q}$.

Now, we present main results on hybrid multisynchronization of delayed CMMNNs as follows.

Theorem 2: The delayed CMMNNs (2) can achieve hybrid multisynchronization and there are $(r+2)^{n}$ hybrid multisynchronization manifolds, if the conditions of corollary 2 hold and there exist a matrix $\hat{\rho}=\operatorname{diag}\left\{\hat{\rho}_{1}, \hat{\rho}_{2}, \ldots, \hat{\rho}_{n}\right\}$, two positive definite matrices $\hat{P}=\operatorname{diag}\left\{\hat{p}_{1}, \hat{p}_{2}, \ldots, \hat{p}_{n}\right\}, \hat{Q}=\operatorname{diag}\left\{\hat{q}_{1}, \hat{q}_{2}, \ldots, \hat{q}_{n}\right\}$ and a positive constant $\delta$, such that

$$
\begin{gathered}
\sum_{j=1}^{n} 2\left(\tilde{\kappa}_{k j}+\tilde{\omega}_{k j}\right) \mu_{j}-\Xi_{k} \varepsilon_{i} \leq 0, \quad k=1, \ldots n, i=1, \ldots, N-1, \\
\hat{p}_{k}\left(\delta-d_{k}-\hat{\rho}_{k}\right)+\frac{1}{2} e^{2 \delta \tau} \hat{q}_{k}\left(l_{k}\right)^{2} \leq 0, \quad k=1,2, \ldots, n,
\end{gathered}
$$

and

$$
W^{T} W\left(\Xi_{j} \Sigma+\hat{\rho}_{j} E_{N}\right) \leq 0, j=1,2, \ldots, n
$$

Proof: See Appendix B.

When $\Omega$ and $\Lambda$ satisfy different conditions, we can get different stable equilibrium states for the master subnetwork of CMMNNs (2). Therefore, we can get corollaries 6 and 7 .

Corollary 6: The delayed CMMNNs (2) can achieve dynamical multisynchronization and there are $(r+2)^{n}$ dynamical multisynchronization manifolds, if the conditions of corollary 1 hold and there exist a matrix $\hat{\rho}=\operatorname{diag}\left\{\hat{\rho}_{1}, \hat{\rho}_{2}, \ldots, \hat{\rho}_{n}\right\}$, two positive definite matrices $\hat{P}=\operatorname{diag}\left\{\hat{p}_{1}, \hat{p}_{2}, \ldots, \hat{p}_{n}\right\}, \hat{Q}=\operatorname{diag}\left\{\hat{q}_{1}, \hat{q}_{2}, \ldots, \hat{q}_{n}\right\}$ and a positive constant $\delta$, such that inequalities (15)-(17) hold. 
Corollary 7: The delayed CMMNNs (2) can achieve static multisynchronization and there are $(r+2)^{n}$ static multisynchronization manifolds, if the conditions of theorem 1 hold and there exist a matrix $\hat{\rho}=\operatorname{diag}\left\{\hat{\rho}_{1}, \hat{\rho}_{2}, \ldots, \hat{\rho}_{n}\right\}$, two

positive definite matrices $\hat{P}=\operatorname{diag}\left\{\hat{p}_{1}, \hat{p}_{2}, \ldots, \hat{p}_{n}\right\}, \hat{Q}=\operatorname{diag}\left\{\hat{q}_{1}, \hat{q}_{2}, \ldots, \hat{q}_{n}\right\}$ and a positive constant $\delta$, such that inequalities (15)-(17) hold.

Remark 6: When the master subnetwork of CMMNNs (2) adds controller, and external inputs of all nodes are set as periodic (or constant) signals, the resulting hybrid multisynchronization will become the dynamical (or static) multisynchronization introduced in [32, 33, 34].

Remark 7: In references [15, 16, 17, 18, 19, 20, 21, 22], [26, 27, 28, 29, 30, 31], $[45,46]$, synchronization of conventional NNs and MNNs under a stable equilibrium state was researched. As discussed previously, multiple stable equilibrium states are very necessary for coupled systems including CMMNNs in some applications [12, 13, 14], [23, 24], [37, 38, 39, 40, 41]. Compared with the above researches $[15,16,17,18,19,20,21,22],[26,27,28,29,30,31],[45,46]$, the highlight of $[32,33,34]$ is that dynamical multisynchronization and static multisynchronization of coupled multistable NNs were addressed under multiple stable equilibrium states. However, these researches $[32,33,34]$ neglect that external inputs of each node may be various in reality. The advantages of this paper are that hybrid multisynchronization of CMMNNs is proposed under multiple stable equilibrium states and the problem mentioned above can be solved by hybrid multisynchronization via considering various external inputs. It is worth emphasizing that dynamical and static multisynchronization can be seen as two particular cases of hybrid multisynchronization. Therefore, the results of this paper are general and meaningful, and extend the existing results.

When PLAF (3) is changed to (12) and memristor model (B) is chosen, we can get the theorem 3 .

Theorem 3: The delayed CMMNNs (2) can achieve hybrid multisynchronization and there are $2^{n}$ hybrid multisynchronization manifolds, if the conditions of corollary 5 hold and there exist a matrix $\hat{\rho}=\operatorname{diag}\left\{\hat{\rho}_{1}, \hat{\rho}_{2}, \ldots, \hat{\rho}_{n}\right\}$, two positive definite matrices $\hat{P}=\operatorname{diag}\left\{\hat{p}_{1}, \hat{p}_{2}, \ldots, \hat{p}_{n}\right\}, \hat{Q}=\operatorname{diag}\left\{\hat{q}_{1}, \hat{q}_{2}, \ldots, \hat{q}_{n}\right\}$ and a 
positive constant $\delta$, such that (15), (17) and

$$
\hat{p}_{k}\left(\delta-d_{k}-\hat{\rho}_{k}\right)+\frac{1}{2} e^{2 \delta \tau} \hat{q}_{k}\left(\beta_{k}^{0}\right)^{2} \leq 0, \quad k=1,2, \ldots, n,
$$

hold.

Proof: From PLAF (12), we have $l_{i}=\beta_{i}^{0}$, the rest of proof is same as theorem 2. So, the detailed proof is omitted here.

When $\Omega$ and $\Lambda$ satisfy different conditions, we can get corollaries 8 and 9 .

Corollary 8: The delayed CMMNNs (2) can achieve dynamical multisynchronization and there are $2^{n}$ dynamical multisynchronization manifolds, if the conditions of corollary 4 hold and there exist a matrix $\hat{\rho}=\operatorname{diag}\left\{\hat{\rho}_{1}, \hat{\rho}_{2}, \ldots, \hat{\rho}_{n}\right\}$, two positive definite matrices $\hat{P}=\operatorname{diag}\left\{\hat{p}_{1}, \hat{p}_{2}, \ldots, \hat{p}_{n}\right\}, \hat{Q}=\operatorname{diag}\left\{\hat{q}_{1}, \hat{q}_{2}, \ldots, \hat{q}_{n}\right\}$ and a positive constant $\delta$, such that inequalities (15), (17) and (18) hold.

Corollary 9: The delayed CMMNNs (2) can achieve static multisynchronization and there are $2^{n}$ static multisynchronization manifolds, if the conditions of corollary 3 hold and there exist a matrix $\hat{\rho}=\operatorname{diag}\left\{\hat{\rho}_{1}, \hat{\rho}_{2}, \ldots, \hat{\rho}_{n}\right\}$, two positive definite matrices $\hat{P}=\operatorname{diag}\left\{\hat{p}_{1}, \hat{p}_{2}, \ldots, \hat{p}_{n}\right\}, \hat{Q}=\operatorname{diag}\left\{\hat{q}_{1}, \hat{q}_{2}, \ldots, \hat{q}_{n}\right\}$ and a positive constant $\delta$, such that inequalities (15), (17) and (18) hold.

Remark 8: In this paper, hybrid, dynamical and static multisynchronization of CMMNNs are addressed with two classes of PLAF and two types of simple memristor models. In practical communication networks, the external inputs of each node may be various. Therefore, compared with dynamical and static multisynchronization, the hybrid multisynchronization is more flexible and practical. 


\section{Simulation}

Example 1. We consider delayed CMMNN which consist of 3 subnetworks as follows.

$$
\left\{\begin{aligned}
\dot{x}_{1}(t) & =-D x_{1}(t)+\Gamma\left(x_{1}(t)\right) f\left(x_{1}(t)\right)+\mathrm{H}\left(x_{1}(t-\tau)\right) \\
\times & f\left(x_{1}(t-\tau)\right)+I_{1}(t)+\sum_{j=1}^{3} \eta_{1 j} \Xi x_{j}(t) \\
+ & \sum_{j=1}^{3} \sigma_{1 j} \Xi \varphi\left(x_{j}(t)-x_{1}(t)\right), \\
\dot{x}_{2}(t) & =-D x_{2}(t)+\Gamma\left(x_{2}(t)\right) f\left(x_{2}(t)\right)+\mathrm{H}\left(x_{2}(t-\tau)\right) \\
& \times f\left(x_{2}(t-\tau)\right)+I_{2}(t)+\sum_{j=1}^{3} \eta_{2 j} \Xi x_{j}(t) \\
+ & \sum_{j=1}^{3} \sigma_{2 j} \Xi \varphi\left(x_{j}(t)-x_{2}(t)\right), \\
\dot{x}_{3}(t) & =-D x_{3}(t)+\Gamma\left(x_{3}(t)\right) f\left(x_{3}(t)\right)+\mathrm{H}\left(x_{3}(t-\tau)\right) \\
& \times f\left(x_{3}(t-\tau)\right)+I_{3}(t),
\end{aligned}\right.
$$

where $x_{i}(t)=\left(x_{i 1}(t), x_{i 2}(t), x_{i 3}(t)\right)^{T}$ for $i=1,2,3$.

Set $f_{j}(w)=\frac{|w+1|-|w-1|}{2}$, then $\mu_{j}=1, \beta_{j}^{0}=1, L_{j}=0, j=1,2,3$.

The other parameters are set as: $\tau_{i j}=0.1, i=1,2,3, j=1,2,3$.

$$
\begin{gathered}
\Gamma\left(x_{i}(t)\right)=\left[\begin{array}{ccc}
\kappa_{11}\left(x_{i 1}(t)\right) & 0 & 0 \\
0 & \kappa_{22}\left(x_{i 2}(t)\right) & 0 \\
0 & 0 & \kappa_{33}\left(x_{i 3}(t)\right)
\end{array}\right], \\
\kappa_{11}\left(x_{i 1}(t)\right)=\left\{\begin{array}{cc}
4.4, & x_{i 1}(t) \leq 0, \\
4.6, & x_{i 1}(t)>0,
\end{array}\right. \\
\kappa_{22}\left(x_{i 2}(t)\right)=\left\{\begin{array}{cc}
4.8, & x_{i 2}(t) \leq 0, \\
3.5, & x_{i 2}(t)>0,
\end{array}\right. \\
\kappa_{33}\left(x_{i 3}(t)\right)=\left\{\begin{array}{cc}
4.0, & x_{i 3}(t) \leq 0, \\
4.5, & x_{i 3}(t)>0,
\end{array}\right. \\
\omega_{11}\left(x_{i 1}(t-\tau)\right)=\left[\begin{array}{c}
\left.\left.\omega_{11}\right)\right) \\
0 \\
0
\end{array}\right.
\end{gathered}
$$




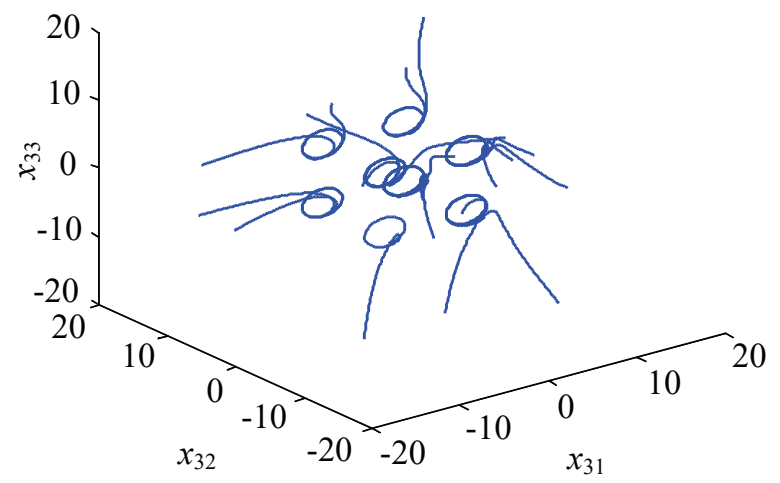

Figure 2: The trajectories of $x_{31}, x_{32}$ and $x_{33}$ with external input vector $I_{3}(t)=$ $(3.2 \sin (t), 2.4,2.8 \cos (t))^{T}$ and 18 random initial values in the interval [-20, 20]. Obviously, there exist 8 hybrid stable equilibrium states for the 3rd subnetwork in $\Re^{3}$

$$
\begin{aligned}
& \omega_{11}\left(x_{i 1}\left(t-\tau_{11}\right)\right)= \begin{cases}0.1, & x_{i 1}\left(t-\tau_{11}\right) \leq 0, \\
0.05, & x_{i 1}\left(t-\tau_{11}\right)>0,\end{cases} \\
& \omega_{22}\left(x_{i 2}\left(t-\tau_{22}\right)\right)= \begin{cases}0.02, & x_{i 2}\left(t-\tau_{22}\right) \leq 0, \\
0.05, & x_{i 2}\left(t-\tau_{22}\right)>0,\end{cases} \\
& \omega_{33}\left(x_{i 3}\left(t-\tau_{33}\right)\right)= \begin{cases}0.08, & x_{i 3}\left(t-\tau_{33}\right) \leq 0, \\
0.04, & x_{i 3}\left(t-\tau_{33}\right)>0,\end{cases}
\end{aligned}
$$

for $i=1,2,3, \delta=0.1, D=\operatorname{diag}\{1,1,1\}$. External input vector $I_{i}(t)=$ $(3.2 \sin (t), 2.4,2.8 \cos (t))^{T}, i=1,2,3$, that is, $\Omega \neq \emptyset, \Lambda \neq \emptyset$. For the $3 \mathrm{rd}$ 325 subnetwork (master subnetwork), we can get that conditions of corollary 5 are satisfied. Therefore, the 3rd subnetwork has 8 hybrid stable equilibrium states in $\Re^{3}$, as shown in Fig. 2.

Let $\hat{Q}=\operatorname{diag}\{0.01,0.01,0.01\}, \Xi=\operatorname{diag}\{10,10,10\}, \hat{\rho}=\operatorname{diag}\{1,1,1\}, \hat{P}=$ $\operatorname{diag}\{1,1,1\} \cdot \sigma_{13}=\sigma_{23}=1$ and $\sigma_{i j}=0$ for $i, j=1,2$, then $\varepsilon_{i}=\sigma_{i 3}-\sum_{j=1}^{2} \sigma_{i j}=$ ${ }_{3 з 0} 1, i=1,2$. We can get that the conditions (15) and (18) are satisfied. 
Set

$$
\Sigma=\left[\eta_{i j}\right]_{3 \times 3}=\left[\begin{array}{ccc}
-2 & 0 & 2 \\
0 & -2 & 2 \\
0 & 0 & 0
\end{array}\right]
$$

then

$$
W^{T} W\left(\Xi_{j} \Sigma+\hat{\rho}_{j} E_{3}\right)=\left[\begin{array}{ccc}
-19 & 0 & 19 \\
0 & -19 & 19 \\
19 & 19 & -38
\end{array}\right] \text {, }
$$

$j=1,2,3$.

The eigenvalues of $W^{T} W\left(\Xi_{j} \Sigma+\hat{\rho}_{j} E_{3}\right)$ are nonpositive:-57, -19, 0 . The condition (17) holds. Thus, CMMNN (19) can achieve hybrid multisynchronization according to the theorem 3. As shown in Fig. 3, there are 18 random initial values for every nerve cell to be tracked. For every nerve cell of the 3rd subnetwork, i.e. $x_{3 j}, j=1,2,3$, there exist 2 trajectories because of 2 corner points of the PLAF. Therefore, there exist 8 hybrid multisynchronization manifolds for the 3rd subnetwork. The hybrid multisynchronization manifolds are $\left.H M S M_{w}(t)\right|_{w=1,2, \ldots, 8}=\left(\left(1^{\#}, 3^{\#}, 5^{\#}\right)^{T},\left(1^{\#}, 3^{\#}, 6^{\#}\right)^{T},\left(1^{\#}, 4^{\#}, 5^{\#}\right)^{T}\right.$, $\left.340 \quad\left(1^{\#}, 4^{\#}, 6^{\#}\right)^{T},\left(2^{\#}, 3^{\#}, 5^{\#}\right)^{T},\left(2^{\#}, 3^{\#}, 6^{\#}\right)^{T},\left(2^{\#}, 4^{\#}, 5^{\#}\right)^{T},\left(2^{\#}, 4^{\#}, 6^{\#}\right)^{T}\right)$.

According to the result of Fig. 3, hybrid multisynchronization of CMMNNs may be applied in some complex areas such as secure communication in multiple networks, obstacle avoidance for robots, formation flying of unmanned air vehicles and so on. For instance, hybrid multisynchronization of CMMNNs is applied in formation flying of unmanned air vehicles. The leaders and the followers of unmanned air vehicles can be simulated by the master subnetwork and the $N-1$ slave subnetworks, respectively. The leaders can be tracked and synchronized by the followers via feedback controllers. For security reason, the leaders usually need to generate multiple flight trajectories according to different initial states (such as fuel loads and device performances) and different external inputs (such as meteorological conditions, human factors). When unmanned air vehicles simulated by CMMNN (19), multiple flight trajectories generated by the leaders can be $\left(1^{\#}, 3^{\#}, 5^{\#}\right)^{T},\left(1^{\#}, 3^{\#}, 6^{\#}\right)^{T},\left(1^{\#}, 4^{\#}, 5^{\#}\right)^{T},\left(1^{\#}, 4^{\#}, 6^{\#}\right)^{T}$, 

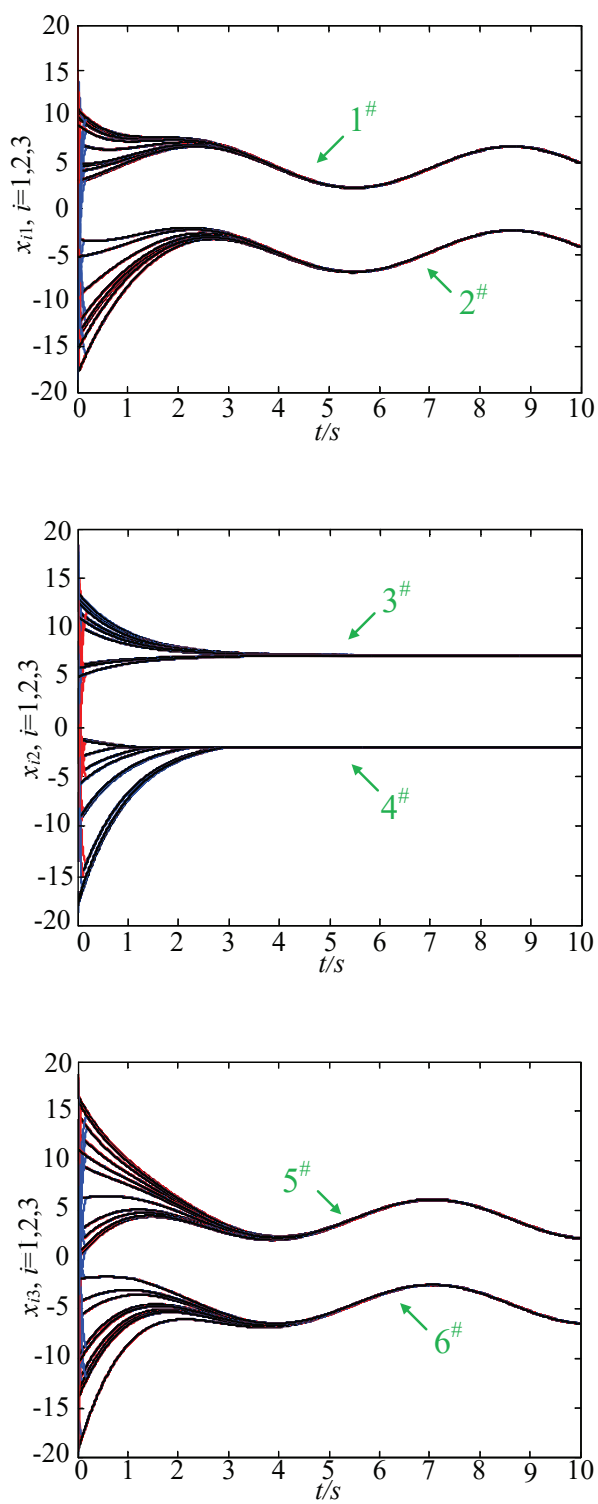

Figure 3: Hybrid multisynchronization of delayed CMMNN (19) with external input vector $I_{i}(t)=(3.2 \sin (t), 2.4,2.8 \cos (t))^{T}$ and 18 random initial values in the interval $[-20,20]$.

$\left(2^{\#}, 3^{\#}, 5^{\#}\right)^{T},\left(2^{\#}, 3^{\#}, 6^{\#}\right)^{T},\left(2^{\#}, 4^{\#}, 5^{\#}\right)^{T},\left(2^{\#}, 4^{\#}, 6^{\#}\right)^{T}$, as shown in Fig. 3.

In this case, we call the synchronization between the leaders and the followers as hybrid multisynchronization of CMMNNs. 
When single initial value is given, delayed CMMNN (19) can achieve exponential synchronization under a stable equilibrium state, as shown in Fig. 4. The hybrid synchronization manifold is $\left(1^{\#}, 4^{\#}, 5^{\#}\right)^{T}$.

Example 2. We consider another delayed CMMNN which consist of 3 subnetworks as follows.

$$
\left\{\begin{aligned}
\dot{x}_{1}(t)= & -D x_{1}(t)+\Gamma\left(x_{1}(t)\right) f\left(x_{1}(t)\right)+\mathrm{H}\left(x_{1}(t-\tau)\right) f\left(x_{1}(t-\tau)\right) \\
& +I_{1}(t)+\sum_{j=1}^{3} \eta_{1 j} \Xi x_{j}(t)+\sum_{j=1}^{3} \sigma_{1 j} \Xi \varphi\left(x_{j}(t)-x_{1}(t)\right), \\
\dot{x}_{2}(t)= & -D x_{2}(t)+\Gamma\left(x_{2}(t)\right) f\left(x_{2}(t)\right)+\mathrm{H}\left(x_{2}(t-\tau)\right) f\left(x_{2}(t-\tau)\right) \\
& +I_{2}(t)+\sum_{j=1}^{3} \eta_{2 j} \Xi x_{j}(t)+\sum_{j=1}^{3} \sigma_{2 j} \Xi \varphi\left(x_{j}(t)-x_{2}(t)\right), \\
\dot{x}_{3}(t)= & -D x_{3}(t)+\Gamma\left(x_{3}(t)\right) f\left(x_{3}(t)\right)+\mathrm{H}\left(x_{3}(t-\tau)\right) f\left(x_{3}(t-\tau)\right) \\
& +I_{3}(t)
\end{aligned}\right.
$$

where $x_{i}(t)=\left(x_{i 1}(t), x_{i 2}(t)\right)^{T}$ for $i=1,2,3$.

Set PLAF as

$$
f_{j}(w)=\left\{\begin{array}{cc}
-4, & (-\infty,-4] \\
10 w+36, & (-4,-3.6) \\
-\frac{2}{7} w-\frac{36}{35}, & {[-3.6,3.4]} \\
10 w-36, & (3.4,4) \\
4, & {[4,+\infty)}
\end{array},\right.
$$

then $\mu_{j}=4, l_{j}=10, L_{j}=2 / 7$, and $f_{j}( \pm 3.6)=0, j=1,2$.

The parameters are set as: $\tau_{i j}=0.1, i=1,2,3, j=1,2$.

$$
\begin{gathered}
\Gamma\left(x_{i}(t)\right)=\left[\begin{array}{cc}
\kappa_{11}\left(x_{i 1}(t)\right) & 0 \\
0 & \kappa_{22}\left(x_{i 2}(t)\right)
\end{array}\right], \\
\kappa_{11}\left(x_{i 1}(t)\right)=\left\{\begin{array}{cc}
4.4, & \left|x_{i 1}(t)\right| \leq 3.6, \\
4.6, & \left|x_{i 1}(t)\right|>3.6,
\end{array}\right. \\
\kappa_{22}\left(x_{i 2}(t)\right)=\left\{\begin{array}{cc}
4.8, & \left|x_{i 2}(t)\right| \leq 3.6, \\
4.0, & \left|x_{i 2}(t)\right|>3.6,
\end{array}\right. \\
\mathrm{H}\left(x_{i}(t-\tau)\right)=\left[\begin{array}{cc}
\omega_{11}\left(x_{i 1}\left(t-\tau_{11}\right)\right) & 0 \\
0 & \omega_{22}\left(x_{i 2}\left(t-\tau_{22}\right)\right)
\end{array}\right],
\end{gathered}
$$



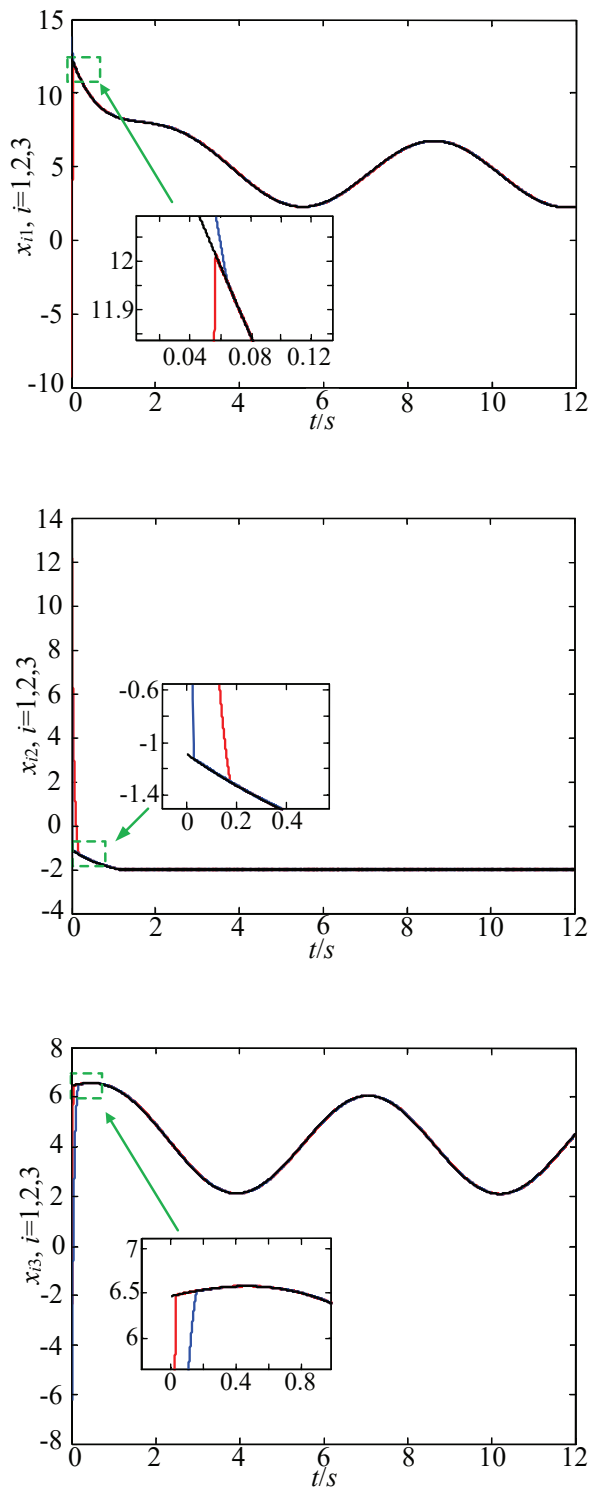

Figure 4: Exponential synchronization of delayed CMMNN (19) under a stable equilibrium state with external input vector $I_{i}(t)=(3.2 \sin (t), 2.4,2.8 \cos (t))^{T}$ and single initial value in the interval $[-10,15]$.

$$
\omega_{11}\left(x_{i 1}\left(t-\tau_{11}\right)\right)=\left\{\begin{array}{cc}
0.1, & \left|x_{i 1}\left(t-\tau_{11}\right)\right| \leq 3.6 \\
0.05, & \left|x_{i 1}\left(t-\tau_{11}\right)\right|>3.6
\end{array}\right.
$$




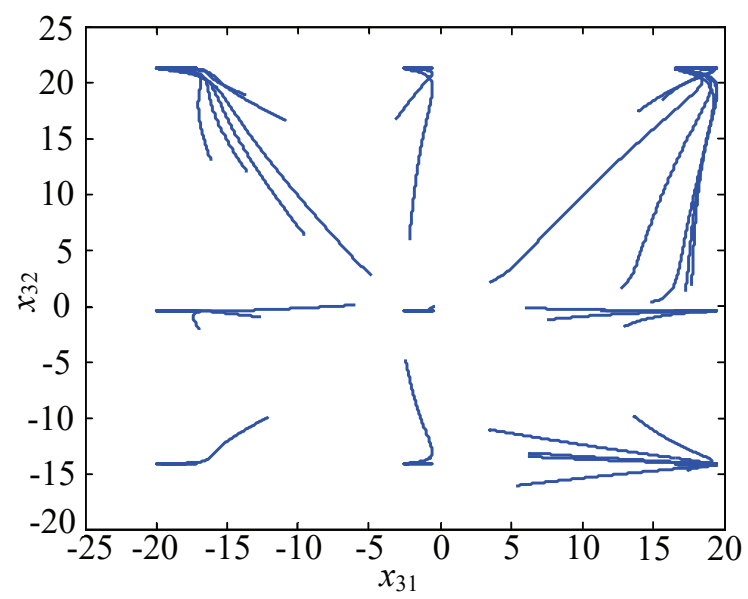

Figure 5: The trajectories of $x_{31}$ and $x_{32}$ with external input vector $I_{3}(t)=(2 \sin (t), 2)^{T}$ and 30 random initial values in the interval $[-20,20]$. There exist 9 hybrid stable equilibrium states for the 3rd subnetwork in $\Re^{2}$

$$
\omega_{22}\left(x_{i 2}\left(t-\tau_{22}\right)\right)= \begin{cases}0.02, & \left|x_{i 2}\left(t-\tau_{22}\right)\right| \leq 3.6 \\ 0.05, & \left|x_{i 2}\left(t-\tau_{22}\right)\right|>3.6\end{cases}
$$

$i=1,2,3, \delta=0.1, D=\operatorname{diag}\{1,1\}$.

External input vector $I_{i}(t)=(2 \sin (t), 2)^{T}, i=1,2,3$, that is, $\Omega \neq \emptyset, \Lambda \neq \emptyset$. For the 3rd subnetwork, we can get that conditions of corollary 2 are satisfied. Therefore, the 3rd subnetwork has 9 hybrid stable equilibrium states in $\Re^{2}$, as shown in Fig 5.

Let $\hat{Q}=\operatorname{diag}\{0.1,0.1\}, \Xi=\operatorname{diag}\{10,10\}, \hat{\rho}=\operatorname{diag}\{0.1,0.1\}, \hat{P}=\operatorname{diag}\{15,15\}$. $\sigma_{i 3}=4$ and $\sigma_{i j}=0$ for $i, j=1,2$, then $\varepsilon_{i}=\sigma_{i 3}-\sum_{j=1}^{2} \sigma_{i j}=4, i=1,2$. Therefore, we can get that inequalities (15) and (16) hold.

Set

$$
\Sigma=\left[\eta_{i j}\right]_{3 \times 3}=\left[\begin{array}{ccc}
-1 & 0 & 1 \\
0 & -1 & 1 \\
0 & 0 & 0
\end{array}\right]
$$


then

$$
W^{T} W\left(\Xi_{j} \Sigma+\hat{\rho}_{j} E_{3}\right)=\left[\begin{array}{ccc}
-9.9 & 0 & 9.9 \\
0 & -9.9 & 9.9 \\
9.9 & 9.9 & -19.8
\end{array}\right]
$$

$370 \quad j=1,2$.

The eigenvalues of $W^{T} W\left(\Xi_{j} \Sigma+\hat{\rho}_{j} E_{3}\right)$ are nonpositive:-29.7, -9.9, 0. The condition (17) holds. Thus, delayed CMMNN (20) can achieve hybrid multisynchronization according to the theorem 2 .

As shown in Fig. 6, CMMNN (20) can achieve hybrid multisynchronization with 30 random initial conditions in the interval $[-20,20]$. Fig. 7 shows the local magnification in the interval $[0,0.1]$ and $[0,0.25]$ with 27 random initial conditions. Red, blue and black lines represent $x_{1 j}, x_{2 j}, x_{3 j}, j=1,2$, respectively. For every nerve cell of the 3 rd subnetwork, i.e. $x_{3 j}, j=1,2$, there exist 3 trajectories because of 4 corner points of the PLAF. Therefore, there exist 9 hybrid multisynchronization manifolds for the 3rd subnetwork. The hybrid multisynchronization manifolds are $\left.H M S M_{w}(t)\right|_{w=1,2, \ldots, 9}=\left(\left(1^{\#}, 4^{\#}\right)^{T},\left(1^{\#}, 5^{\#}\right)^{T}\right.$, $\left.\left(1^{\#}, 6^{\#}\right)^{T},\left(2^{\#}, 4^{\#}\right)^{T},\left(2^{\#}, 5^{\#}\right)^{T},\left(2^{\#}, 6^{\#}\right)^{T},\left(3^{\#}, 4^{\#}\right)^{T},\left(3^{\#}, 5^{\#}\right)^{T},\left(3^{\#}, 6^{\#}\right)^{T}\right)$.

When single initial value is given, delayed CMMNN (20) can achieve exponential synchronization under a stable equilibrium state, as shown in Fig. 8.

\section{Conclusion}

This paper builds delayed CMMNNs and investigates multistability of delayed CMMNNs with continuous PLAF owning $2 r+2$ corner points. Sufficient conditions certify that there exist $(r+2)^{n}$ exponentially stable equilibrium points, stable periodic orbits or hybrid stable equilibrium states. Then, we propose hybrid multisynchronization based on the structure of delayed CMMNNs for the first time and can obtain $(r+2)^{n}$ hybrid multisynchronization manifolds. Hybrid multisynchronization can solve the problem that dynamical and static multisynchronization $[32,33,34]$ cannot take into consideration various 

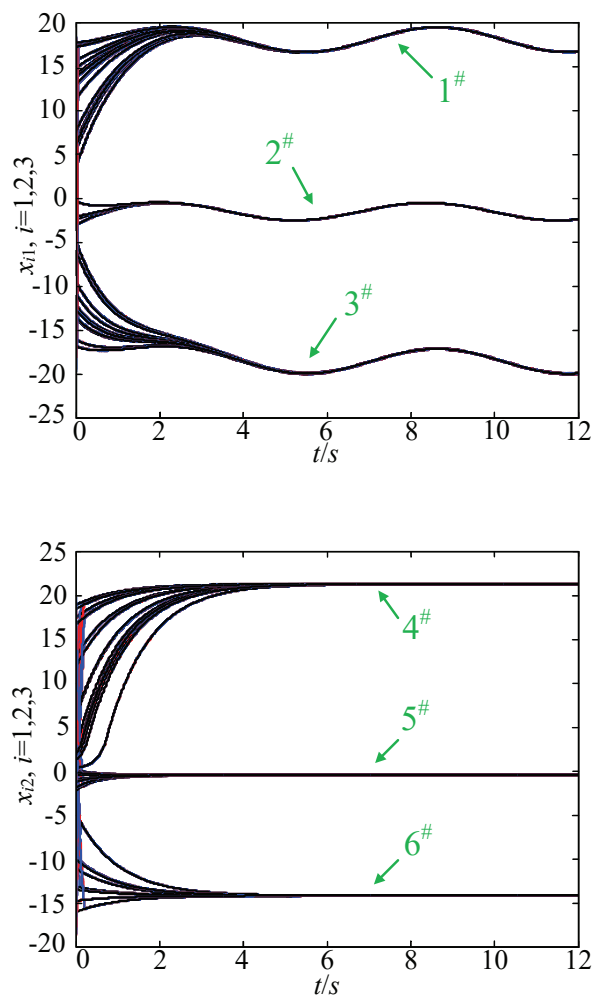

Figure 6: Hybrid multisynchronization of delayed CMMNN (20) with external input vector $I_{i}(t)=(2 \sin (t), 2)^{T}$ and 30 random initial values in the interval $[-20,20]$.

external inputs. Moreover, hybrid, dynamical and static multisynchronization of CMMNNs are addressed with two classes of PLAF and two types of simple memristor models. Compared with dynamical and static multisynchronization, the hybrid multisynchronization is more flexible and practical. Therefore, the results of this paper are general and meaningful, and extend the existing results.

In the future research, hybrid multisynchronization of CMMNNs can be achieved via different feedback control schemes, such as pinning control, adaptive control and so on. Moreover, further investigation can focus on the robust hybrid multisynchronization of CMMNNs with parameter perturbations. 

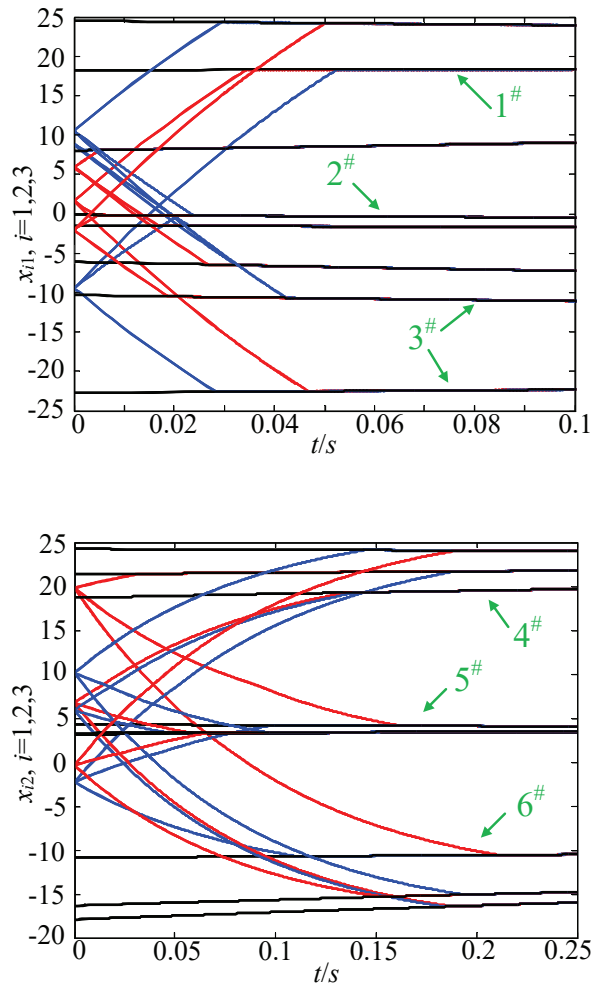

Figure 7: The local magnification in the interval $[0,0.1]$ and $[0,0.25]$ with 27 random initial conditions.

\section{A. Proof of Theorem 1}

First, denote

$$
\begin{aligned}
\Phi= & \left\{\prod_{i=1}^{n}\left(-\infty, p_{i}^{0}\right]^{\lambda_{i}^{1}} \times\left(p_{i}^{0}, q_{i}^{0}\right)^{0} \times\left[q_{i}^{0}, p_{i}^{1}\right]^{\lambda_{i}^{2}}\right. \\
& \times \cdots \times\left(p_{i}^{r}, q_{i}^{r}\right)^{0} \times\left[q_{i}^{r},+\infty\right)^{\lambda_{i}^{r+2}}, \\
& \left(\lambda_{i}^{1}, \lambda_{i}^{2}, \cdots, \lambda_{i}^{r+2}\right)=(1,0, \cdots, 0) \text { or }(0,1, \cdots, 0) \\
& \text { or } \cdots \text { or }(0,0, \cdots, 1)\} .
\end{aligned}
$$

We will prove that $(r+2)^{n}$ exponentially stable equilibrium points locate in $\Phi$ in three steps.

Step 1: We will prove that there exist $(r+2)^{n}$ equilibrium points located in $\Phi$. 

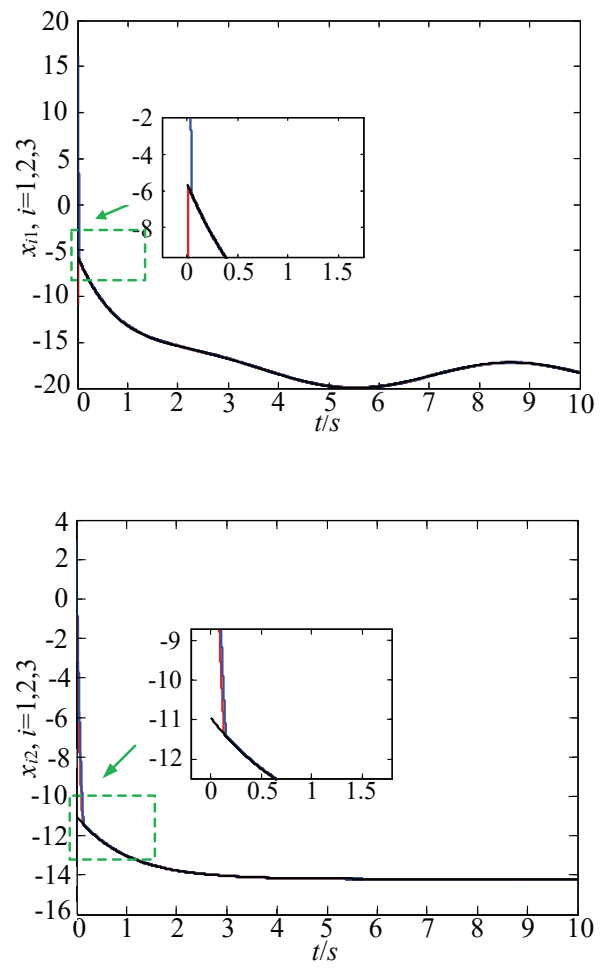

Figure 8: Exponential synchronization of delayed CMMNN (20) under a stable equilibrium state with external input vector $I_{i}(t)=(2 \sin (t), 2)^{T}$ and single initial value in the interval $[-25,20]$.

From $\Lambda=\vartheta$, we can get that every element in set $\vartheta$ is constant. So, by isolated MNN (1), the master subnetwork of CMMNNs (2) can be rewritten as:

$$
\dot{x}(t)=-D x(t)+\hat{\Gamma}(x(t)) f(x(t))+\hat{\mathrm{H}}(x(t-\tau)) f(x(t-\tau))+I,
$$

where $I=\left(I_{1}, I_{2}, \ldots, I_{n}\right)^{T}$.

Take an arbitrary region $\tilde{\Phi}$ from set $\Phi$, for arbitrary $\left(x_{1}, x_{2}, \ldots, x_{n}\right)^{T} \in \tilde{\Phi}$, fix $x_{1}, \ldots, x_{i-1}, x_{i+1}, \ldots, x_{n}$ except $x_{i}(t)$, and define

$$
\begin{gathered}
G_{i}\left(x_{i}(t)\right)=-d_{i} x_{i}(t)+\hat{\kappa}_{i i}\left(x_{i}(t)\right) f_{i}\left(x_{i}(t)\right)+\sum_{j=1, j \neq i}^{n} \hat{\kappa}_{i j}\left(x_{j}(t)\right) f_{j}\left(x_{j}(t)\right) \\
+\sum_{j=1}^{n} \hat{\omega}_{i j}\left(x_{j}\left(t-\tau_{i j}\right)\right) f_{j}\left(x_{j}\left(t-\tau_{i j}\right)\right)+I_{i} .
\end{gathered}
$$


Then, there exist three cases that will be discussed.

Case 1. When $x_{i}(t) \in\left(-\infty, p_{i}^{0}\right]$, we can have

$$
\begin{aligned}
G_{i}\left(p_{i}^{0}\right) & =-d_{i} p_{i}^{0}+\hat{\kappa}_{i i}\left(p_{i}^{0}\right) f_{i}\left(p_{i}^{0}\right)+\sum_{j=1, j \neq i}^{n} \hat{\kappa}_{i j}\left(x_{j}(t)\right) \\
& \times f_{j}\left(x_{j}(t)\right)+\sum_{j=1}^{n} \hat{\omega}_{i j}\left(x_{j}\left(t-\tau_{i j}\right)\right) f_{j}\left(x_{j}\left(t-\tau_{i j}\right)\right)+I_{i} \\
\leq & -d_{i} p_{i}^{0}+\max \left\{\breve{\kappa}_{i i} f_{i}\left(p_{i}^{0}\right), \bar{\kappa}_{i i} f_{i}\left(p_{i}^{0}\right)\right\} \\
& +\sum_{j=1, j \neq i}^{n} \max \left\{\breve{\kappa}_{i j} u_{j}, \bar{\kappa}_{i j} u_{j}, \breve{\kappa}_{i j} v_{j}, \bar{\kappa}_{i j} v_{j}\right\} \\
& +\sum_{j=1}^{n} \max \left\{\breve{\omega}_{i j} u_{j}, \bar{\omega}_{i j} u_{j}, \breve{\omega}_{i j} v_{j}, \bar{\omega}_{i j} v_{j}\right\}+I_{i}<0 .
\end{aligned}
$$

Noticing the continuity of function $G_{i}(x)$ and $\lim _{x \rightarrow-\infty} G_{i}(x)=+\infty$, so there exists a point $\bar{x}_{i} \in\left(-\infty, p_{i}^{0}\right]$ such that $G_{i}\left(\bar{x}_{i}\right)=0$.

Case 2. When $x_{i}(t) \in\left(q_{i}^{c}, p_{i}^{c+1}\right], c=0,1, \ldots, r-1$, we can have

$$
\begin{aligned}
& G_{i}\left(q_{i}^{c}\right) \geq-d_{i} q_{i}^{c}+\min \left\{\breve{\kappa}_{i i} f_{i}\left(q_{i}^{c}\right), \bar{\kappa}_{i i} f_{i}\left(q_{i}^{c}\right)\right\} \\
& \quad+\sum_{j=1, j \neq i}^{n} \min \left\{\breve{\kappa}_{i j} u_{j}, \bar{\kappa}_{i j} u_{j}, \breve{\kappa}_{i j} v_{j}, \bar{\kappa}_{i j} v_{j}\right\} \\
& \quad+\sum_{j=1}^{n} \min \left\{\breve{\omega}_{i j} u_{j}, \bar{\omega}_{i j} u_{j}, \breve{\omega}_{i j} v_{j}, \bar{\omega}_{i j} v_{j}\right\}+I_{i}>0 .
\end{aligned}
$$

and

$$
\begin{aligned}
& G_{i}\left(p_{i}^{c+1}\right) \leq-d_{i} p_{i}^{c+1}+\max \left\{\breve{\kappa}_{i i} f_{i}\left(p_{i}^{c+1}\right), \bar{\kappa}_{i i} f_{i}\left(p_{i}^{c+1}\right)\right\} \\
& \quad+\sum_{j=1, j \neq i}^{n} \max \left\{\breve{\kappa}_{i j} u_{j}, \bar{\kappa}_{i j} u_{j}, \breve{\kappa}_{i j} v_{j}, \bar{\kappa}_{i j} v_{j}\right\} \\
& \quad+\sum_{j=1}^{n} \max \left\{\breve{\omega}_{i j} u_{j}, \bar{\omega}_{i j} u_{j}, \breve{\omega}_{i j} v_{j}, \bar{\omega}_{i j} v_{j}\right\}+I_{i}<0 .
\end{aligned}
$$

Therefore, there exists a point $\bar{x}_{i} \in\left(q_{i}^{c}, p_{i}^{c+1}\right]$ such that $G_{i}\left(\bar{x}_{i}\right)=0, c=$ $0,1, \ldots, r-1$.

Case 3. When $x_{i}(t) \in\left(q_{i}^{r},+\infty\right]$, we can have

$$
\begin{aligned}
& G_{i}\left(q_{i}^{r}\right) \geq-d_{i} q_{i}^{r}+\min \left\{\breve{\kappa}_{i i} f_{i}\left(q_{i}^{r}\right), \bar{\kappa}_{i i} f_{i}\left(q_{i}^{r}\right)\right\} \\
& \quad+\sum_{j=1, j \neq i}^{n} \min \left\{\breve{\kappa}_{i j} u_{j}, \bar{\kappa}_{i j} u_{j}, \breve{\kappa}_{i j} v_{j}, \bar{\kappa}_{i j} v_{j}\right\} \\
& \quad+\sum_{j=1}^{n} \min \left\{\breve{\omega}_{i j} u_{j}, \bar{\omega}_{i j} u_{j}, \breve{\omega}_{i j} v_{j}, \bar{\omega}_{i j} v_{j}\right\}+I_{i}>0 .
\end{aligned}
$$

415

Combining $\lim _{x \rightarrow+\infty} G_{i}(x)=-\infty$, we can find a point $\bar{x}_{i} \in\left(q_{i}^{r},+\infty\right]$ such that $G_{i}\left(\bar{x}_{i}\right)=0$. 
As set $\Phi$ consists of $(r+2)^{n}$ parts, we can get that there exist $(r+2)^{n}$ equilibrium points for the master subnetwork of CMMNNs (2) in $\Re^{n}$ with PLAF (3) and memristor model (A) by Brouwer's fixed point theorem.

Step 2: Set $x_{i}(t)$ is the solution for the $i$ th node of the master subnetwork of CMMNNs (2) with respect to initial condition $x_{i}(0) \in \tilde{\Phi}$. Then, for all $t \geq 0$, we assert that $x_{i}(t)$ will stay in $\tilde{\Phi}$. If this is false, then there exist three cases to be discussed.

Case 1. When $x_{i}(0) \in\left(-\infty, p_{i}^{0}\right]$, then there exists $t^{(1)} \geq 0$ such that $x_{i}\left(t^{(1)}\right)=p_{i}^{0}, \dot{x}_{i}\left(t^{(1)}\right)>0$, and $x_{i}(t) \leq p_{i}^{0}$ for $0 \leq t \leq t^{(1)}$. Actually,

$$
\begin{aligned}
& \dot{x}_{i}\left(t^{(1)}\right)=-d_{i} x_{i}\left(t^{(1)}\right)+\hat{\kappa}_{i i}\left(x_{i}\left(t^{(1)}\right)\right) f_{i}\left(x_{i}\left(t^{(1)}\right)\right) \\
& \quad+\sum_{j=1, j \neq i}^{n} \hat{\kappa}_{i j}\left(x_{j}\left(t^{(1)}\right)\right) f_{j}\left(x_{j}\left(t^{(1)}\right)\right) \\
& \quad+\sum_{j=1}^{n} \hat{\omega}_{i j}\left(x_{j}\left(t^{(1)}-\tau_{i j}\right)\right) f_{j}\left(x_{j}\left(t^{(1)}-\tau_{i j}\right)\right)+I_{i} \\
& \quad \leq-d_{i} p_{i}^{0}+\max \left\{\breve{\kappa}_{i i} f_{i}\left(p_{i}^{0}\right), \bar{\kappa}_{i i} f_{i}\left(p_{i}^{0}\right)\right\} \\
& \quad+\sum_{j=1, j \neq i}^{n} \max \left\{\breve{\kappa}_{i j} u_{j}, \bar{\kappa}_{i j} u_{j}, \breve{\kappa}_{i j} v_{j}, \bar{\kappa}_{i j} v_{j}\right\} \\
& \quad+\sum_{j=1}^{n} \max \left\{\breve{\omega}_{i j} u_{j}, \bar{\omega}_{i j} u_{j}, \breve{\omega}_{i j} v_{j}, \bar{\omega}_{i j} v_{j}\right\}+I_{i}<0,
\end{aligned}
$$

so, it is contradictory.

Case 2. When $x_{i}(0) \in\left[q_{i}^{c}, p_{i}^{c+1}\right], c=0,1, \ldots, r-1$, then there exists $t^{(2)} \geq 0$ such that

(1) $x_{i}\left(t^{(2)}\right)=q_{i}^{c}, \dot{x}_{i}\left(t^{(2)}\right)<0, x_{i}(t) \in\left[q_{i}^{c}, p_{i}^{c+1}\right], 0 \leq t \leq t^{(2)}$;

or $(2) x_{i}\left(t^{(2)}\right)=p_{i}^{c+1}, \dot{x}_{i}\left(t^{(2)}\right)>0, x_{i}(t) \in\left[q_{i}^{c}, p_{i}^{c+1}\right], 0 \leq t \leq t^{(2)}$.

For case (1), we have

$$
\begin{aligned}
& \dot{x}_{i}\left(t^{(2)}\right) \geq-d_{i} q_{i}^{c}+\min \left\{\breve{\kappa}_{i i} f_{i}\left(q_{i}^{c}\right), \bar{\kappa}_{i i} f_{i}\left(q_{i}^{c}\right)\right\} \\
& \quad+\sum_{j=1, j \neq i}^{n} \min \left\{\breve{\kappa}_{i j} u_{j}, \bar{\kappa}_{i j} u_{j}, \breve{\kappa}_{i j} v_{j}, \bar{\kappa}_{i j} v_{j}\right\} \\
& \quad+\sum_{j=1}^{n} \min \left\{\breve{\omega}_{i j} u_{j}, \bar{\omega}_{i j} u_{j}, \breve{\omega}_{i j} v_{j}, \bar{\omega}_{i j} v_{j}\right\}+I_{i}>0,
\end{aligned}
$$

this is contradictory. Similarly, the case (2) can be overturned.

Case 3. When $x_{i}(0) \in\left[q_{i}^{r},+\infty\right)$, then there exist $t^{(3)} \geq 0$ such that $x_{i}\left(t^{(3)}\right)=$ 
$q_{i}^{r}, \dot{x}_{i}\left(t^{(3)}\right)<0$, and $x_{i}(t) \geq q_{i}^{r}, 0 \leq t \leq t^{(3)}$. But,

$$
\begin{aligned}
& \dot{x}\left(t^{(3)}\right) \geq-d_{i} q_{i}^{r}+\min \left\{\breve{\kappa}_{i i} f_{i}\left(q_{i}^{r}\right), \bar{\kappa}_{i i} f_{i}\left(q_{i}^{r}\right)\right\} \\
& \quad+\sum_{j=1, j \neq i}^{n} \min \left\{\breve{\kappa}_{i j} u_{j}, \bar{\kappa}_{i j} u_{j}, \breve{\kappa}_{i j} v_{j}, \bar{\kappa}_{i j} v_{j}\right\} \\
& \quad+\sum_{j=1}^{n} \min \left\{\breve{\omega}_{i j} u_{j}, \bar{\omega}_{i j} u_{j}, \breve{\omega}_{i j} v_{j}, \bar{\omega}_{i j} v_{j}\right\}+I_{i}>0,
\end{aligned}
$$

which is a contradiction.

Through the above analysis, we can get that for all $t \geq 0, x_{i}(t)$ will stay in $\tilde{\Phi}$.

Step 3: we will prove that the $(r+2)^{n}$ equilibrium points are exponentially stable.

Denote a function $U_{i}(\zeta)=d_{i}-\zeta-\sum_{j=1}^{n} \tilde{\kappa}_{i j} L_{j}-\sum_{j=1}^{n} e^{\zeta \tau_{i j}} \tilde{\omega}_{i j} L_{j}$, then $U_{i}(0)>0$, and there exists a sufficiently small positive constant $\theta$, such that $U_{i}(\theta)>0$ for any $i(i=1,2, \ldots, n)$. Taking an arbitrary region $\tilde{\Phi}$ from the set $\Phi$, we can find an equilibrium point $\bar{x}_{i}$ in region $\tilde{\Phi}$. Set $y_{i}(t)=x_{i}(t)-\bar{x}_{i}, i=1, \ldots, n$. In terms of theory of differential inclusion, we can get

$$
\begin{aligned}
\dot{y}_{i}(t) & \in-d_{i} y_{i}(t)+\sum_{j=1}^{n}\left\{c o\left[\kappa_{i j}\left(x_{j}(t)\right)\right] f_{j}\left(x_{j}(t)\right)\right. \\
& \left.-c o\left[\kappa_{i j}\left(\bar{x}_{j}\right)\right] f_{j}\left(\bar{x}_{j}\right)\right\}+\sum_{j=1}^{n}\left\{c o\left[\omega_{i j}\left(x_{j}\left(t-\tau_{i j}\right)\right)\right]\right. \\
& \left.\times f_{j}\left(x_{j}\left(t-\tau_{i j}\right)\right)-c o\left[\omega_{i j}\left(\bar{x}_{j}\right)\right] f_{j}\left(\bar{x}_{j}\right)\right\} .
\end{aligned}
$$

$x_{i}(t), i=1,2, \ldots, n$ stay in $\tilde{\Phi}$, which means that Lipschitz condition for $f_{i}(s)$ in remark 2 can be rewritten as

$$
\left|f_{i}(\wedge)-f_{i}(\vee)\right| \leq L_{i}|\wedge-\vee|, i=1,2, \ldots, n,
$$

for any $\wedge, \vee \in \Re$.

Therefore, from lemma 1, we can get

$$
\left|c o\left[\kappa_{i j}\left(x_{j}(t)\right)\right] f_{j}\left(x_{j}(t)\right)-c o\left[\kappa_{i j}\left(\bar{x}_{j}\right)\right] f_{j}\left(\bar{x}_{j}\right)\right| \leq \tilde{\kappa}_{i j} L_{j}\left|y_{j}(t)\right|,
$$

and

$$
\left|c o\left[\omega_{i j}\left(x_{j}\left(t-\tau_{i j}\right)\right)\right] f_{j}\left(x_{j}\left(t-\tau_{i j}\right)\right)-c o\left[\omega_{i j}\left(\bar{x}_{j}\right)\right] f_{j}\left(\bar{x}_{j}\right)\right| \leq \tilde{\omega}_{i j} L_{j}\left|y_{j}\left(t-\tau_{i j}\right)\right| .
$$


Hence,

$$
\frac{d}{d t}\left|y_{i}(t)\right| \leq-d_{i}\left|y_{i}(t)\right|+\sum_{j=1}^{n} \tilde{\kappa}_{i j} L_{j}\left|y_{j}(t)\right|+\sum_{j=1}^{n} \tilde{\omega}_{i j} L_{j}\left|y_{j}\left(t-\tau_{i j}\right)\right| .
$$

Set $z_{\ell}(t)=e^{\theta t}\left|y_{\ell}(t)\right|, \ell=1,2, \ldots, n$ and $\max _{1 \leq i \leq n}\left\{\left|x_{i}(0)-\bar{x}_{i}\right|\right\}>0$. We can get $z_{\ell}(0) \leq \max _{1 \leq i \leq n}\left\{\left|x_{i}(0)-\bar{x}_{i}\right|\right\}, \ell=1,2, \ldots, n$

Then, we will prove the following inequality by contradiction:

$$
z_{\ell}(t) \leq \max _{1 \leq i \leq n}\left\{\left|x_{i}(0)-\bar{x}_{i}\right|\right\}, \quad t>0, \ell=1,2, \ldots, n
$$

Let $\hat{\mathrm{O}}=\max _{1 \leq i \leq n}\left\{\left|x_{i}(0)-\bar{x}_{i}\right|\right\}$. Suppose (22) is invalid, then we can find a $k \in\{1, \ldots, n\}$ and $t_{1}$ for the first time $z_{k}\left(t_{1}\right)=\hat{\mathrm{O}}, \dot{z}_{k}\left(t_{1}\right)>0, z_{k}(t) \leq \hat{\mathrm{O}}$, $t \in\left[0, t_{1}\right) ; z_{i}(t) \leq \hat{\mathrm{O}}, t \in\left[0, t_{1}\right], i=1,2, \ldots, n, i \neq k$. Actually,

$$
\begin{aligned}
\dot{z}_{k}\left(t_{1}\right) & =\theta e^{\theta t_{1}}\left|y_{k}\left(t_{1}\right)\right|+e^{\theta t_{1}} \frac{d}{d t}\left|y_{k}\left(t_{1}\right)\right| \\
\leq & \theta z_{k}\left(t_{1}\right)-d_{k} z_{k}\left(t_{1}\right)+\sum_{j=1}^{n} \tilde{\kappa}_{k j} L_{j} z_{j}\left(t_{1}\right) \\
+ & \sum_{j=1}^{n} e^{\theta \tau_{k j}} \tilde{\omega}_{k j} L_{j} z_{j}\left(t_{1}-\tau_{k j}\right) \\
\leq & \left\{\theta-d_{k}+\sum_{j=1}^{n} \tilde{\kappa}_{k j} L_{j}+\sum_{j=1}^{n} e^{\theta \tau_{k j}} \tilde{\omega}_{k j} L_{j}\right\} \\
& \times \max _{1 \leq i \leq n}\left\{\left|x_{i}(0)-\bar{x}_{i}\right|\right\} \\
= & -U_{k}(\theta) \max _{1 \leq i \leq n}\left\{\left|x_{i}(0)-\bar{x}_{i}\right|\right\}<0
\end{aligned}
$$

Thus, this is contradictory, namely, (22) holds. Therefore, we can have that $\left|x_{\ell}(t)-\bar{x}_{\ell}\right| \leq e^{-\theta t} \max _{1 \leq i \leq n}\left\{\left|x_{i}(0)-\bar{x}_{i}\right|\right\}, \ell=1,2, \ldots, n$. In other words, equilib440

rium point $\bar{x}_{i}$ in $\tilde{\Phi}$ is exponentially stable. Further, the master subnetwork of CMMNNs $(2)$ can find $(r+2)^{n}$ exponentially stable equilibrium points in $\Re^{n}$ with PLAF (3) and memristor model (A). The proof is finished.

\section{B. Proof of Theorem 2}

We consider a Lyapunov functional as

$$
V(t)=e(t)^{T} \tilde{P} e(t) e^{2 \delta t}+\int_{t-\tau}^{t} f(x(s))^{T} \tilde{W}^{T} \tilde{Q} \tilde{W} f(x(s)) e^{2 \delta(s+\tau)} d s
$$


Calculating the derivative of $V(t)$, we get

$$
\begin{aligned}
& D^{+} V(t)=2 \delta e^{2 \delta t} e(t)^{T} \tilde{P} e(t)+2 e^{2 \delta t} e(t)^{T} \tilde{P} \dot{e}(t) \\
& \quad+e^{2 \delta(t+\tau)} f(x(t))^{T} \tilde{W}^{T} \tilde{Q} \tilde{W} f(x(t)) \\
& \quad-e^{2 \delta t} f(x(t-\tau))^{T} \tilde{W}^{T} \tilde{Q} \tilde{W} f(x(t-\tau)) \\
& \quad=2 \delta e^{2 \delta t} x(t)^{T} \tilde{W}^{T} \tilde{P} \tilde{W} x(t) \\
& \quad+2 e^{2 \delta t} x(t)^{T} \tilde{W}^{T} \tilde{P} \tilde{W}\left\{-\left(E_{N} \otimes D\right) x(t)\right. \\
& \quad+\left(E_{N} \otimes \Gamma(x(t))\right) f(x(t)) \\
& \quad+\left(E_{N} \otimes \mathrm{H}(x(t-\tau))\right) f(x(t-\tau))+\bar{I}(t) \\
& \left.\quad+(\Sigma \otimes \Xi) x(t)+\Theta+\left(E_{N} \otimes \hat{\rho}\right) x(t)-\left(E_{N} \otimes \hat{\rho}\right) x(t)\right\} \\
& \quad+e^{2 \delta(t+\tau)} f(x(t))^{T} \tilde{W}^{T} \tilde{Q} \tilde{W} f(x(t)) \\
& \quad-e^{2 \delta t} f(x(t-\tau))^{T} \tilde{W}^{T} \tilde{Q} \tilde{W} f(x(t-\tau))
\end{aligned}
$$

Now, we analyze each item of $D^{+} V(t)$.

$$
\begin{aligned}
& 2 e^{2 \delta t} x(t)^{T} \tilde{W}^{T} \tilde{P} \tilde{W} \Theta=2 e^{2 \delta t} \sum_{i=1}^{N-1} \sum_{k=1}^{n} \hat{p}_{k} \Xi_{k}\left(x_{i k}(t)\right. \\
& \left.\quad-x_{N k}(t)\right) \times \sum_{j=1}^{N} \sigma_{i j} \operatorname{sgn}\left(x_{j k}-x_{i k}\right) \\
& =2 e^{2 \delta t} \sum_{i=1}^{N-1} \sum_{k=1}^{n} \hat{p}_{k} \Xi_{k}\left\{\left|x_{i k}(t)-x_{N k}(t)\right|\left(-\sigma_{i N}\right)\right. \\
& \left.\quad+\left(x_{i k}(t)-x_{N k}(t)\right) \sum_{j=1}^{N-1} \sigma_{i j} \operatorname{sgn}\left(x_{j k}-x_{i k}\right)\right\} \\
& \leq 2 e^{2 \delta t} \sum_{i=1}^{N-1} \sum_{k=1}^{n} \hat{p}_{k} \Xi_{k}\left|x_{i k}(t)-x_{N k}(t)\right|\left\{-\sigma_{i N}+\sum_{j=1}^{N-1} \sigma_{i j}\right\} \\
& =-2 e^{2 \delta t} \sum_{i=1}^{N-1} \sum_{k=1}^{n} \hat{p}_{k} \Xi_{k} \varepsilon_{i}\left|e_{i k}(t)\right| .
\end{aligned}
$$


and

$$
\begin{aligned}
& 2 \delta e^{2 \delta t} x(t)^{T} \tilde{W}^{T} \tilde{P} \tilde{W} x(t)+2 e^{2 \delta t} x(t)^{T} \tilde{W}^{T} \tilde{P} \tilde{W} \\
& \times\left\{-\left(E_{N} \otimes D\right) x(t)+\left(E_{N} \otimes \Gamma(x(t))\right) f(x(t))\right. \\
&\left.+\left(E_{N} \otimes \mathrm{H}(x(t-\tau))\right) f(x(t-\tau))-\left(E_{N} \otimes \hat{\rho}\right) x(t)\right\} \\
&= 2 \delta e^{2 \delta t} x(t)^{T} \tilde{W}^{T} \tilde{P} \tilde{W} x(t)-2 e^{2 \delta t} x(t)^{T} \tilde{W}^{T} \tilde{P}\left(E_{N-1} \otimes D\right) \tilde{W} x(t) \\
&-2 e^{2 \delta t} x(t)^{T} \tilde{W}^{T} \tilde{P}\left(E_{N-1} \otimes \hat{\rho}\right) \tilde{W} x(t) \\
&+2 e^{2 \delta t} x(t)^{T} \tilde{W}^{T} \tilde{P}\left(E_{N-1} \otimes \Gamma(x(t))\right) \tilde{W} f(x(t)) \\
&+2 e^{2 \delta t} x(t)^{T} \tilde{W}^{T} \tilde{P}\left(E_{N-1} \otimes \mathrm{H}(x(t-\tau))\right) \tilde{W} f(x(t-\tau)) \\
&= 2 e^{2 \delta t} x(t)^{T} \tilde{W}^{T} \tilde{P}\left[\left(\delta E_{N-1} \otimes E_{n}\right)-\left(E_{N-1} \otimes D\right)-\left(E_{N-1} \otimes \hat{\rho}\right)\right] \\
& \times \tilde{W} x(t)+2 e^{2 \delta t} x(t)^{T} \tilde{W}^{T} \tilde{P}\left(E_{N-1} \otimes \Gamma(x(t))\right) \tilde{W} f(x(t)) \\
&+2 e^{2 \delta t} x(t)^{T} \tilde{W}^{T} \tilde{P}\left(E_{N-1} \otimes \mathrm{H}(x(t-\tau))\right) \tilde{W} f(x(t-\tau))
\end{aligned}
$$

Combining remark 2, we can get

$$
\begin{aligned}
2 \delta e^{2 \delta t} x(t)^{T} \tilde{W}^{T} \tilde{P} \tilde{W} x(t)+2 e^{2 \delta t} x(t)^{T} \tilde{W}^{T} \tilde{P} \tilde{W} \\
\quad \times\left\{-\left(E_{N} \otimes D\right) x(t)+\left(E_{N} \otimes \Gamma(x(t))\right) f(x(t))\right. \\
\left.\quad+\left(E_{N} \otimes \mathrm{H}(x(t-\tau))\right) f(x(t-\tau))-\left(E_{N} \otimes \hat{\rho}\right) x(t)\right\} \\
=2 e^{2 \delta t} \sum_{i=1}^{N-1} \sum_{k=1}^{n} \hat{p}_{k}\left(x_{i k}(t)-x_{N k}(t)\right)^{2}\left(\delta-d_{k}-\hat{\rho}_{k}\right) \\
\quad+2 e^{2 \delta t} \sum_{i=1}^{N-1} \sum_{k=1}^{n} \hat{p}_{k}\left(x_{i k}(t)-x_{N k}(t)\right) \sum_{j=1}^{n}\left[\kappa_{k j}\left(x_{j}(t)\right)\right. \\
\quad \times\left(f_{j}\left(x_{i j}(t)\right)-f_{j}\left(x_{N j}(t)\right)\right) \\
\left.\quad+\omega_{k j}\left(x_{j}\left(t-\tau_{k j}\right)\right)\left(f_{j}\left(x_{i j}\left(t-\tau_{i j}\right)\right)-f_{j}\left(x_{N j}\left(t-\tau_{N j}\right)\right)\right)\right] \\
\leq 2 e^{2 \delta t} \sum_{i=1}^{N-1} \sum_{k=1}^{n} \hat{p}_{k}\left(e_{i k}(t)\right)^{2}\left(\delta-d_{k}-\hat{\rho}_{k}\right) \\
\quad+2 e^{2 \delta t} \sum_{i=1}^{N-1} \sum_{k=1}^{n} \hat{p}_{k}\left|e_{i k}(t)\right| \sum_{j=1}^{n} 2\left(\tilde{\kappa}_{k j}+\tilde{\omega}_{k j}\right) \mu_{j} .
\end{aligned}
$$

Obviously, $\tilde{W} \bar{I}(t)=0$, and

$$
\begin{gathered}
-e^{2 \delta t} f(x(t-\tau))^{T} \tilde{W}^{T} \tilde{Q} \tilde{W} f(x(t-\tau)) \\
=-e^{2 \delta t} \sum_{i=1}^{N-1} \sum_{k=1}^{n}\left(f_{k}\left(x_{i k}\left(t-\tau_{i k}\right)\right)\right. \\
\left.-f_{k}\left(x_{N k}\left(t-\tau_{N k}\right)\right)\right)^{2} \hat{q}_{k} \leq 0 .
\end{gathered}
$$


Therefore,

$$
\begin{aligned}
& e^{2 \delta(t+\tau)} f(x(t))^{T} \tilde{W}^{T} \tilde{Q} \tilde{W} f(x(t))-e^{2 \delta t} f(x(t-\tau))^{T} \tilde{W}^{T} \tilde{Q} \tilde{W} f(x(t-\tau)) \\
& \leq e^{2 \delta(t+\tau)} \sum_{i=1}^{N-1} \sum_{k=1}^{n}\left(f_{k}\left(x_{i k}(t)\right)-f_{k}\left(x_{N k}(t)\right)\right) \hat{q}_{k}\left(f_{k}\left(x_{i k}(t)\right)-f_{k}\left(x_{N k}(t)\right)\right) \\
& \leq e^{2 \delta(t+\tau)} \sum_{i=1}^{N-1} \sum_{k=1}^{n} \hat{q}_{k}\left(l_{k}\right)^{2}\left(x_{i k}(t)-x_{N k}(t)\right)^{2} \\
& =e^{2 \delta(t+\tau)} \sum_{i=1}^{N-1} \sum_{k=1}^{n} \hat{q}_{k}\left(l_{k}\right)^{2}\left(e_{i k}(t)\right)^{2}
\end{aligned}
$$

Moreover,

$$
\begin{aligned}
& 2 e^{2 \delta t} x(t)^{T} \tilde{W}^{T} \tilde{P} \tilde{W}\left\{(\Sigma \otimes \Xi) x(t)+\left(E_{N} \otimes \hat{\rho}\right) x(t)\right\} \\
& =2 e^{2 \delta t} \sum_{j=1}^{n} \hat{p}_{j} \tilde{x}_{j}(t)^{T} W^{T} W\left(\Xi_{j} \Sigma+\hat{\rho}_{j} E_{N}\right) \tilde{x}_{j}(t) \leq 0
\end{aligned}
$$

where $\tilde{x}_{j}(t)=\left(x_{1 j}(t), x_{2 j}(t), \ldots, x_{N j}(t)\right)^{T}$.

Therefore,

$$
\begin{aligned}
D^{+} V & (t)=2 e^{2 \delta t} \sum_{i=1}^{N-1} \sum_{k=1}^{n} \hat{p}_{k}\left(e_{i k}(t)\right)^{2}\left(\delta-d_{k}-\hat{\rho}_{k}\right) \\
& +2 e^{2 \delta t} \sum_{i=1}^{N-1} \sum_{k=1}^{n} \hat{p}_{k}\left|e_{i k}(t)\right| \sum_{j=1}^{n} 2\left(\tilde{\kappa}_{k j}+\tilde{\omega}_{k j}\right) \mu_{j} \\
& -2 e^{2 \delta t} \sum_{i=1}^{N-1} \sum_{k=1}^{n} \hat{p}_{k} \Xi_{k} \varepsilon_{i}\left|e_{i k}(t)\right|+e^{2 \delta(t+\tau)} \sum_{i=1}^{N-1} \sum_{k=1}^{n} \hat{q}_{k} \\
& \times\left(l_{k}\right)^{2}\left(e_{i k}(t)\right)^{2}+2 e^{2 \delta t} \sum_{j=1}^{n} \hat{p}_{j} \tilde{x}_{j}(t)^{T} W^{T} W \\
& \times\left(\Xi_{j} \Sigma+\hat{\rho}_{j} E_{N}\right) \tilde{x}_{j}(t) \\
& =2 e^{2 \delta t} \sum_{i=1}^{N-1} \sum_{k=1}^{n}\left(e_{i k}(t)\right)^{2}\left[\hat{p}_{k}\left(\delta-d_{k}-\hat{\rho}_{k}\right)\right. \\
& \left.+\frac{1}{2} e^{2 \delta \tau} \hat{q}_{k}\left(l_{k}\right)^{2}\right]+2 e^{2 \delta t} \sum_{i=1}^{N-1} \sum_{k=1}^{n} \hat{p}_{k}\left|e_{i k}(t)\right| \\
& \times\left[\sum_{j=1}^{n} 2\left(\tilde{\kappa}_{k j}+\tilde{\omega}_{k j}\right) \mu_{j}-\Xi_{k} \varepsilon_{i}\right] \\
+ & 2 e^{2 \delta t} \sum_{j=1}^{n} \hat{p}_{j} \tilde{x}_{j}(t)^{T} W^{T} W\left(\Xi_{j} \Sigma+\hat{\rho}_{j} E_{N}\right) \tilde{x}_{j}(t) \leq 0
\end{aligned}
$$

From Lyapunov functional, we can get

$$
V(t) \geq \hat{p}_{\min } e^{2 \delta t} \sum_{i=1}^{N-1} \sum_{j=1}^{n}\left(e_{i j}(t)\right)^{2},
$$

${ }_{445}$ where $\hat{p}_{\min }=\min _{1 \leq i \leq n}\left\{\hat{p}_{i}\right\}$. 
Then,

$$
\begin{aligned}
& \left\|x_{i}(t)-x_{N}(t)\right\|=\left\|e_{i}(t)\right\| \\
& \leq \sqrt{\hat{p}_{\min }^{-1} e^{-2 \delta t} V(t)} \leq \hat{p}_{\min }^{-0.5} \sqrt{V(0)} e^{-\delta t},
\end{aligned}
$$

for $i=1, \ldots, N-1, j=1, \ldots, n$.

Therefore, as time $t \rightarrow+\infty, x_{i j}(t) \rightarrow x_{N j}(t)$ for any given initial values, where $i=1, \ldots, N-1, j=1, \ldots, n$.

For the master subnetwork of CMMNNs (2), the conditions of corollary 2 hold, that means master subnetwork of CMMNNs (2) can own $(r+2)^{n}$ hybrid stable equilibrium states in $\Re^{n}$ with PLAF (3) and memristor model (A). Therefore, there are $(r+2)^{n}$ hybrid multisynchronization manifolds. The proof is completed.

\section{References}

[1] L. Chua, Memristor-the missing circuit element, IEEE Transactions on Circuit Theory 18 (5) (1971) 507-519.

[2] D. Strukov, G. Snider, D. Stewart, R. Williams, The missing memristor found, Nature 453 (2008) 80-83.

[3] L. Zhou, C. Wang, L. Zhou, Generating hyperchaotic multi-wing attractor in a 4d memristive circuit, Nonlinear Dynamics 85 (4) (2016) 2653-2663.

[4] L. Zhou, C. Wang, L. Zhou, A novel no-equilibrium hyperchaotic multiwing system via introducing memristor, International Journal of Circuit Theory and Applications 46 (1) (2018) 84-98.

[5] C. Wang, X. Liu, H. Xia, Multi-piecewise quadratic nonlinearity memristor and its 2n-scroll and 2n+1-scroll chaotic attractors system, Chaos 27 (3) (2017) 033114-1-033114-12.

[6] L. Zhou, C. Wang, L. Zhou, Generating four-wing hyperchaotic attractor and two-wing, three-wing, and four-wing chaotic attractors in $4 \mathrm{~d}$ memristive system, International Journal of Bifurcation and Chaos 27 (2) (2017) 1750027-1-1750027-14. 
[7] S. Jo, T. Chang, I. Ebong, B. Bhadviya, P. Mazumder, W. Lu, Nanoscale memristor device as synapse in neuromorphic systems, Nano Letters 10 (4) (2010) 1297-1301.

[8] H. Kim, M. P. Sah, C. Yang, T. Roska, L. Chua, Memristor bridge synapse, Proceedings of the IEEE 100 (6) (2012) 2061-2070.

[9] J. Yang, L. Wang, Y. Wang, T. Guo, A novel memristive hopfield neural network with application in associative memory, Neurocomputing 227 (2017) 142-148.

[10] X. Hu, S. Duan, G. Chen, L. Chen, Modeling affections with memristorbased associative memory neural networks, Neurocomputing 223 (2017) $129-137$.

[11] S. Duan, X. Hu, L. Wang, S. Gao, C. Li, Hybrid memristor/rtd structurebased cellular neural networks with applications in image processing, Neural Computing and Applications 25 (2) (2014) 291-296.

[12] Z. Guo, J. Wang, Y. Zheng, Global exponential dissipativity and stabilization of memristor-based recurrent neural networks with time-varying delays, Neural Networks 48 (6) (2013) 158-172.

[13] A. Wu, J. Zhang, Multistability of memristive neural networks with timevarying delays, Complexity 21 (1) (2015) 177-186.

[14] X. Nie, W. Zheng, J. Cao, Coexistence and local $\mu$-stability of multiple equilibrium points for memristive neural networks with nonmonotonic piecewise linear activation functions and unbounded time-varying delays, Neural Networks 84 (2016) 172-180.

[15] S. Wen, G. Bao, Z. Zeng, Y. Chen, T. Huang, Global exponential synchronization of memristor-based recurrent neural networks with time-varying delays, Neural Networks 48 (2013) 195-203. 
[16] A. Wu, S. Wen, Z. Zeng, Synchronization control of a class of memristorbased recurrent neural networks, Information Sciences 183 (1) (2012) 106116.

[17] A. Wu, S. Wen, Z. Zeng, Anti-synchronization control of a class of memristive recurrent neural networks, Communications in Nonlinear Science and Numerical Simulation 18 (2) (2013) 373-385.

[18] M. Zheng, L. Li, H. Peng, J. Xiao, Y. Yang, H. Zhao, Finite-time projective synchronization of memristor-based delay fractional-order neural networks, Nonlinear Dynamics 89 (4) (2017) 2641-2655.

[19] L. Wang, Y. Shen, Q. Yin, G. Zhang, Adaptive synchronization of memristor-based neural networks with time-varying delays, IEEE Transactions on Neural Networks and Learning Systems 26 (9) (2015) 2033-2042.

[20] H. Bao, J. Park, J. Cao, Adaptive synchronization of fractional-order memristor-based neural networks with time delay, Nonlinear Dynamics 82 (3) (2015) 1343-1354.

[21] H. Bao, J. Cao, J. Kurths, State estimation of fractional-order delayed memristive neural networks, Nonlinear Dynamics 94 (2) (2018) 1215-1225.

[22] H. Bao, J. Cao, J. Kurths, A. Alsaedi, B. Ahmad, H $\infty$ state estimation of stochastic memristor-based neural networks with time-varying delays, Neural Networks 99 (2018) 79-91.

[23] T. Guo, L. Wang, M. Zhou, S. Duan, A multi-layer memristive recurrent neural network for solving static and dynamic image associative memory, Neurocomputingdoi: 10.1016/j.neucom.2018.12.056.

[24] X. Hu, G. Feng, S. Duan, L. Liu, A memristive multilayer cellular neural network with applications to image processing, IEEE Transactions on Neural Networks and Learning Systems 28 (8) (2017) 1889-1901. 
[25] M. Rubenstein, A. Cornejo, R. Nagpal, Programmable self-assembly in a thousand-robot swarm, Science 345 (2014) 795-799.

[26] G. Wang, Y. Shen, Q. Yin, Exponential synchronization of coupled memristive neural networks via pinning control, Chinese Physics B 22 (5) (2013) 203-212.

[27] W. Zhang, C. Li, T. Huang, X. He, Synchronization of memristor-based coupling recurrent neural networks with time-varying delays and impulses, IEEE Transactions on Neural Networks and Learning Systems 26 (12) (2015) 3308-3313.

[28] N. Li, J. Cao, Lag synchronization of memristor-based coupled neural networks via $\omega$-measure, IEEE Transactions on Neural Networks and Learning Systems 27 (3) (2016) 686-697.

[29] H. Bao, J. Park, J. Cao, Exponential synchronization of coupled stochastic memristor-based neural networks with time-varying probabilistic delay coupling and impulsive delay, IEEE Transactions on Neural Networks and Learning Systems 27 (1) (2016) 190-201.

[30] X. Yang, J. Cao, J. Lu, Synchronization of randomly coupled neural networks with markovian jumping and time-delay, IEEE Transactions on Circuits and Systems I: Regular Papers 60 (2) (2013) 363-376.

[31] L. Li, J. Cao, Cluster synchronization in an array of coupled stochastic delayed neural networks via pinning control, Neurocomputing 74 (5) (2011) 846-856.

[32] Y. W. Wang, W. Yang, J. W. Xiao, Z. G. Zeng, Impulsive multisynchronization of coupled multistable neural networks with time-varying delay, IEEE Transactions on Neural Networks and Learning Systems 28 (7) (2017) 1560-1571.

[33] J. E. Zhang, Multisynchronization for coupled multistable fractional-order neural networks via impulsive control, Complexity 2017 (2017) 1-10. 
[34] X. Lv, X. Li, J. Cao, M. Perc, Dynamical and static multisynchronization of coupled multistable neural networks via impulsive control, IEEE Transactions on Neural Networks and Learning Systems 29 (12) (2018) $6062-6072$.

[35] S. Yang, Z. Guo, J. Wang, Global synchronization of multiple recurrent neural networks with time delays via impulsive interactions, IEEE Transactions on Neural Networks and Learning Systems 28 (7) (2017) 1657-1667.

[36] D. Angeli, J. E. Ferrell, E. D. Sontag, Detection of multistability, bifurcations, and hysteresis in a large class of biological positive-feedback systems, Proceedings of the National Academy of Sciences 101 (7) (2004) 1822-1827.

[37] E. Kaslik, S. Sivasundaram, Impulsive hybrid discrete-time hopfield neural networks with delays and multistability analysis, Neural Networks 24 (4) (2011) 370-377.

[38] W. H. Chen, S. Luo, X. Lu, Multistability in a class of stochastic delayed hopfield neural networks, Neural Networks 68 (2015) 52-61.

[39] Z. Zeng, T. Huang, W. Zheng, Multistability of recurrent neural networks with time-varying delays and the piecewise linear activation function, IEEE Transactions on Neural Networks 21 (8) (2010) 1371-1377.

[40] W. Yang, Y. W. Wang, Z. G. Zeng, D. F. Zheng, Multistability of discretetime delayed cohen-grossberg neural networks with second-order synaptic connectivity, Neurocomputing 164 (2015) 252-261.

[41] L. Wang, W. Lu, T. Chen, Coexistence and local stability of multiple equilibria in neural networks with piecewise linear nondecreasing activation functions, Neural Networks 23 (2) (2010) 189-200.

575 [42] R. Erichsen, L. G. Brunnet, Multistability in networks of hindmarsh-rose neurons, Physical Review E 78 (6) (2008) 061917. 
[43] H. Weimerskirch, J. Martin, Y. Clerquin, P. Alexandre, S. Jiraskova, Energy saving in flight formation, Nature 413 (2001) 697-698.

[44] L. Shanmugam, P. Mani, R. Rajan, Y. H. Joo, Adaptive synchronization of reaction-diffusion neural networks and its application to secure communication, IEEE Transactions on Cybernetics 112doi:10.1109/tcyb.2018.2877410.

[45] J. Cao, Y. Wan, Matrix measure strategies for stability and synchronization of inertial bam neural network with time delays, Neural Networks 53 (2014) $165-172$.

[46] Y. Xia, Z. Yang, M. Han, Lag synchronization of unknown chaotic delayed yang-yang-type fuzzy neural networks with noise perturbation based on adaptive control and parameter identification, IEEE Transactions on Neural Networks 20 (7) (2009) 1165-1180. 